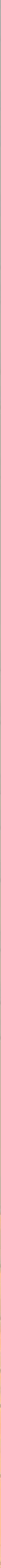




\title{
Modularity of the concave composition generating function
}

\author{
George E. Andrews, Robert C. Rhoades and Sander P. Zwegers
}

A composition of an integer constrained to have decreasing then increasing parts is called concave. We prove that the generating function for the number of concave compositions, denoted $v(q)$, is a mixed mock modular form in a more general sense than is typically used.

We relate $v(q)$ to generating functions studied in connection with "Moonshine of the Mathieu group" and the smallest parts of a partition. We demonstrate this connection in four different ways. We use the elliptic and modular properties of Appell sums as well as $q$-series manipulations and holomorphic projection.

As an application of the modularity results, we give an asymptotic expansion for the number of concave compositions of $n$. For comparison, we give an asymptotic expansion for the number of concave compositions of $n$ with strictly decreasing and increasing parts, the generating function of which is related to a false theta function rather than a mock theta function.

\section{Introduction}

A composition of an integer $n$ is a sum of positive integers adding to $n$, in which order matters. The study of compositions has a long history dating back to MacMahon [1893]. The book of Heubach and Mansour [2010] contains more on the history of compositions. It is natural to impose restrictions on the ascents or descents of consecutive parts of a composition. For instance, compositions with no ascents correspond to integer partitions.

A concave composition of $n$ is a sum of integers of the form

$$
\sum_{i=1}^{L} a_{i}+c+\sum_{i=1}^{R} b_{i}=n
$$

where $a_{1} \geq \cdots \geq a_{L}>c<b_{1} \leq \cdots \leq b_{R}$, where $c \geq 0$ is the central part of the composition. Let $V(n)$ be the number of concave compositions of $n$. For

MSC2010: primary 05A17; secondary 11P82, 11F03.

Keywords: concave composition, partition, unimodal sequences, mock theta function, mixed mock modular form. 
example, $V(3)=13$ since $\{0,3\},\{3,0\},\{0,1,2\},\{2,1,0\},\{0,1,1,1\},\{1,1,1,0\}$, $\{1,2\},\{2,1\},\{1,0,2\},\{2,0,1\},\{1,0,1,1\},\{1,1,0,1\}$, and $\{3\}$ are all concave sequences. In [Andrews 2011] it was shown that the generating function for concave compositions with further restrictions is related to statistics for spiral self-avoiding random walks as well as other partition problems.

Standard techniques show that the generating function for the sequence $\{V(n)\}_{n=0}^{\infty}$ is given by

$$
v(q):=\sum_{n=0}^{\infty} V(n) q^{n}=\sum_{n=0}^{\infty} \frac{q^{n}}{\left(q^{n+1} ; q\right)_{\infty}^{2}}
$$

where $(x)_{n}=(x ; q)_{n}:=\prod_{j=0}^{n-1}\left(1-x q^{j}\right)$ and $(x)_{\infty}=(x ; q)_{\infty}:=\prod_{j=0}^{\infty}\left(1-x q^{j}\right)$; see [Andrews 2013]. We establish the modularity properties of $v(q)$ as a mixed mock modular form.

Theorem 1.1. Let $q=e^{2 \pi i \tau}$ and $\tau \in \mathbb{H}$. Define $f(\tau)=q(q)_{\infty}^{3} v(q)$ and $\widehat{f}(\tau):=f(\tau)-\frac{i}{2} \eta(\tau)^{3} \int_{-\bar{\tau}}^{i \infty} \frac{\eta(z)^{3}}{(-i(z+\tau))^{\frac{1}{2}}} d z+\frac{\sqrt{3}}{2 \pi i} \eta(\tau) \int_{-\bar{\tau}}^{i \infty} \frac{\eta(z)}{(-i(z+\tau))^{\frac{3}{2}}} d z$ where the Dedekind $\eta$-function is given by $\eta(\tau)=q^{1 / 24}(q)_{\infty}$. The function $\hat{f}$ transforms as a modular form of weight 2 for $\mathrm{SL}_{2}(\mathbb{Z})$.

Remark. Theorem 1.1 was used by Bryson, Ono, Pitman and the second author [Bryson et al. 2012] to show that the modular form $f(\tau) /(q)_{\infty}=q(q)_{\infty}^{2} v(q)$ produces a quantum modular form. The $q$-hypergeometric series defining $q(q)_{\infty}^{2} v(q)$ was shown to be dual to Kontsevich's strange function

$$
F(q):=\sum_{n=0}^{\infty}(q ; q)_{n}
$$

which is defined only when $q$ is a root of unity and was studied by Zagier [2001].

Following Zagier [2009], a mock theta function of weight $k \in\left\{\frac{1}{2}, \frac{3}{2}\right\}$ is a $q$-series $H(q)=\sum_{n=0}^{\infty} a_{n} q^{n}$ such that there exists a rational number $\lambda$ and a unary theta function of weight $2-k, g(\tau)=\sum_{n \in \mathbb{Q}^{+}} b_{n} q^{n}$, where $q=e^{2 \pi i \tau}=e^{2 \pi i(x+i y)}$, such that $h(\tau)=q^{\lambda} H(q)+g^{*}(\tau)$ is a nonholomorphic modular form of weight $k$, where

$$
g^{*}(\tau)=(i / 2)^{k-1} \sum_{n \in \mathbb{Q}^{+}} n^{k-1} \bar{b}_{n} \Gamma(1-k, 4 \pi n y) q^{-n}
$$

and $\Gamma(k, t)=\int_{t}^{\infty} u^{k-1} e^{-u} d u$ is the incomplete gamma function. Such a nonholomorphic modular form is called a harmonic weak Maass form (see Section 2 for a definition and Ono's surveys [2008; 2009] for history). The theta function $g$ is called the shadow of the mock theta function $H$. 
In [Andrews 2013] the following identity is established, which is crucial in establishing Theorem 1.1. We have

$$
v(q)=q^{-1}\left(v_{1}(q)+v_{2}(q)+v_{3}(q)\right)
$$

where

$$
\begin{aligned}
& v_{1}(q):=\frac{1}{(q)_{\infty}^{3}}\left(\sum_{n \neq 0} \frac{(-1)^{n+1} q^{3 n(n+1) / 2}}{\left(1-q^{n}\right)^{2}}-3 \sum_{n=1}^{\infty} \frac{q^{n}}{\left(1-q^{n}\right)^{2}}+\frac{1}{12}\right), \\
& v_{2}(q):=\frac{1}{(q)_{\infty}^{3}}\left(\sum_{n \neq 0} \frac{(-1)^{n+1} n q^{n(n+1) / 2}}{1-q^{n}}-\frac{1}{4}-2 \sum_{n=1}^{\infty} \frac{q^{n}}{\left(1+q^{n}\right)^{2}}\right), \\
& v_{3}(q):=\frac{1}{(q)_{\infty}^{3}}\left(\frac{1}{6}+2 \sum_{n=1}^{\infty} q^{n}\left(\frac{1}{\left(1+q^{n}\right)^{2}}+\frac{1}{\left(1-q^{n}\right)^{2}}\right)\right) .
\end{aligned}
$$

Theorem 1.1 may be recast in the following terms.

Theorem 1.2. With $q=e^{2 \pi i z}$ and $z \in \mathbb{W}$ we have:

(1) $q^{-1 / 24}(q)_{\infty}^{2} v_{1}(q)$ is a mock theta function of weight $\frac{3}{2}$ with shadow proportional to $\eta(z)$. Consequentially, $v_{1}(q)$ is a mixed mock modular form.

(2) $q^{-1 / 8} v_{2}(q)$ is a mock theta function of weight $\frac{1}{2}$ with shadow proportional to $\eta(z)^{3}$.

(3) $q^{-1 / 8} v_{3}(q)$ is a modular form of weight $\frac{1}{2}$.

Remark. Theorem 4.1 gives the level and shadow for each of the corresponding harmonic weak Maass form.

The mock theta function $v_{2}(q)$ has appeared in a number of interesting places. For example, it arises in the work of Eguchi, Ooguri, and Tachikawa [Eguchi et al. 2011] and Cheng [2010] concerned with the character table of the Mathieu group $M_{24}$ and "Moonshine of the Mathieu group". It also appears in [Malmendier and Ono 2012], which deals with Donaldson invariants of $\mathbb{C} P^{2}$. In that work $v_{2}(q)$ arises in a different form, which is equivalent to the following identity. Moreover, the claim for $v_{2}(q)$ in Theorem 1.2 follows from the next theorem and the results in [Zwegers 2002].

Theorem 1.3. For $|q|<1$ we have

$$
\begin{aligned}
\tilde{F}(q):=\frac{1}{(q)_{\infty}(-q)_{\infty}^{2}} & \sum_{n \in \mathbb{Z}} \frac{q^{n(n+1) / 2}}{1+q^{n}} \\
& =\frac{1}{(q)_{\infty}^{3}}\left(\frac{1}{2}+4 \sum_{n \geq 1} \frac{q^{n}}{\left(1+q^{n}\right)^{2}}-2 \sum_{n \neq 0} \frac{(-1)^{n+1} n q^{n(n+1) / 2}}{1-q^{n}}\right) .
\end{aligned}
$$


We give three different ways of obtaining this identity. Each proof relies on different symmetries of Appell sums. The first uses an elliptic transformation property and Taylor expansions of the Jacobi theta function. The second proof uses the modular properties of the Appell sums. The third is via $q$-series manipulations. Finally, we sketch a connection with the holomorphic projection construction for mock modular forms.

Remark. Theorem 11.1 gives an analogous result for the Appell sum in the definition of $v_{1}(q)$. Moreover, it relates $v_{1}(q)$ to the smallest parts generating function studied in [Andrews 2008].

There is a convenient graphical representation of a composition, where each part is represented by a vertical column of boxes; for example,

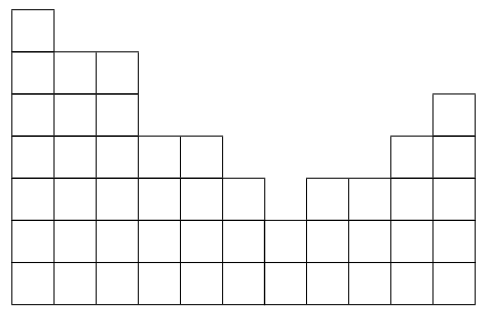

represents a concave composition of 47. Considering each composition of $n$, possibly from a restricted subset, it is natural to ask about the average limiting behavior of the graphical representation as $n \rightarrow \infty$. For example, there is a great deal of literature about the limiting shape of integer partitions; see, for instance, [Fristedt 1993; Pittel 1997; Vershik 1995].

Properties of the typical representation are often studied via probabilistic models. However, when the generating functions are modular forms, very strong theorems can be proved for the statistics of interest. For instance, the modularity of the generating function for the number of partitions and the circle method yield the following asymptotic expansion for the number of partitions of $n$, denoted $p(n)$ :

$$
p(n)=\frac{2 \sqrt{3}}{(24 n-1)} \exp \left(\frac{\pi}{6} \sqrt{24 n-1}\right)\left(1-\frac{6}{\pi \sqrt{24 n-1}}+O\left(\frac{1}{n^{T}}\right)\right)
$$

for any $T \geq 0$.

As an application of the modular properties of $v(q)$ we give an asymptotic expansion for $V(n)$. Since the generating function is a mixed mock modular form, the circle method may be applied to obtain asymptotics for $V(n)$. Care must be taken to deal with contributions from the holomorphic Fourier series as well as the nonholomorphic period integrals. We use methods of Bringmann and Mahlburg [2011; 2013] for dealing with these difficulties.

Theorem 1.4. For any $T \geq 1$ as $n \rightarrow \infty$, we have the asymptotic expansion 


$$
\begin{aligned}
V(n)=\frac{2 \sqrt{6}}{\left(12 n+\frac{21}{2}\right)^{3 / 4}} \exp \left(\frac{\pi}{3} \sqrt{12 n+\frac{21}{2}}\right) \\
\times\left(\sum_{t=1}^{T} \frac{(2 t-1) ! ! 3^{t} \alpha_{t}}{2^{2 t} \pi^{t}} \frac{1}{\left(12 n+\frac{21}{2}\right)^{t / 2}}+O\left(\frac{1}{n^{(T+1) / 2}}\right)\right)
\end{aligned}
$$

where $\alpha_{t}$ are defined by

$$
\sum_{k=1}^{\infty} \alpha_{k} x^{2 k}:=\exp \left(\sqrt{1-2 x^{2}}-1+x^{2}\right) x \frac{\sinh \left(\frac{2 \pi x}{3}\right)}{\cosh (\pi x)} .
$$

In particular, $\alpha_{1}=2 \pi / 3$.

Remark. It is possible to obtain an asymptotic with a polynomial error term; see [Bringmann and Mahlburg 2011; 2013; Rhoades 2012].

Remark. A concave composition corresponds to a triple $(\lambda, \mu, c)$ where $\lambda$ and $\mu$ are partitions, and $c \geq 0$ is an integer strictly smaller than the smallest parts in $\lambda$ and $\mu$, such that $n=|\lambda|+|\mu|+c$. Fristedt's results [1993] imply that partitions of size $n$ have a part of size 1 with probability roughly equal to $1-(\pi / \sqrt{6 n})$. We expect at least one of the partitions $\mu$ or $\lambda$ to have size not much smaller than $n / 2$. That partition will almost surely contain a part of size 1 . Thus we expect $c=0$ for most triples. Therefore, we expect that $V(n)$ will agree to leading order with the asymptotic for the number of pairs of partitions $(\mu, \lambda)$ with $|\mu|+|\lambda|=n$. Standard circle method calculations show this number is

$$
\frac{\sqrt{6}}{(12 n-1)^{5 / 4}} \exp \left(\frac{\pi}{3} \sqrt{12 n-1}\right)\left(1+O\left(\frac{1}{\sqrt{n}}\right)\right) \text {. }
$$

As expected this agrees with the leading order asymptotic of $V(n)$.

For comparison we introduce the notion of strongly concave compositions. A strongly concave composition of $n$ is a sum of integers of the form

$$
\sum_{i=1}^{L} a_{i}+c+\sum_{i=1}^{R} b_{i}=n
$$

where $a_{1}>\cdots>a_{L}>c<b_{1}<\cdots<b_{R}$ and where $c \geq 0$. Let $V_{d}(n)$ be the number of strongly concave compositions of $n$. We have

$$
\begin{aligned}
v_{d}(q) & :=\sum_{n=0}^{\infty} V_{d}(n) q^{n}=\sum_{n=0}^{\infty} q^{n}\left(-q^{n+1}\right)_{\infty}\left(-q^{n+1}\right)_{\infty} \\
& =-\sum_{n=0}^{\infty}(-1)^{n} q^{n(n+1) / 2}+2(-q)_{\infty}^{2} \sum_{n \geq 0}\left(\frac{-12}{n}\right) q^{\left(n^{2}-1\right) / 24}
\end{aligned}
$$


where the second equality follows from standard techniques and the third equality is established in [Andrews 2013] and $(\dot{*})$ is the Kronecker symbol. The function $\sum_{n \geq 0}(-12 / n) q^{\left(n^{2}-1\right) / 24}$ is called a partial theta function. The asymptotic behavior of the partial theta function is discussed in Section 10 and used to derive the following theorem for $V_{d}(n)$.

Theorem 1.5. For any $T \geq 0$ as $n \rightarrow \infty$, we have

$$
\begin{aligned}
V_{d}(n)=\frac{\sqrt{3}}{(24 n+2)^{3 / 4}} & \exp \left(\frac{\pi}{6} \sqrt{24 n+2}\right) \\
\times & \left(\sum_{m=0}^{T} \frac{(-1)^{m}}{2^{2 m} m !(24 n+2)^{m / 2}} \gamma(m)+O\left(\frac{1}{n^{(T+1) / 2}}\right)\right),
\end{aligned}
$$

where

$$
\gamma(m)=\sum_{a+b=m}\left(\begin{array}{c}
m \\
a
\end{array}\right) L(-2 a)\left(\frac{\pi}{3}\right)^{a-b} p(b, a)
$$

with $p(b, a)=\prod_{j=0}^{b}\left(4(a-1)^{2}-j^{2}\right)$ and

$$
L(-r)=-\frac{6^{r}}{r+1}\left(B_{r+1}\left(\frac{1}{6}\right)-B_{r+1}\left(\frac{5}{6}\right)\right),
$$

where $B_{r}(x)$ is the $r$-th Bernoulli polynomial.

Remark. Let $\operatorname{sm}(\lambda)$ be the smallest part in the partition $\lambda$. Strongly concave compositions are characterized by a pair of partitions into distinct parts $(\lambda, \mu)$ with $\operatorname{sm}(\lambda) \neq \operatorname{sm}(\mu)$. Let $\tilde{u}(n)$ be the number of pairs of partitions into distinct parts with sizes summing to $n$. Therefore, we expect the asymptotic of

$$
V_{d}(n) \sim(1-\operatorname{Prob}\{\operatorname{sm}(\lambda)=\operatorname{sm}(\mu):|\lambda|+|\mu|=n\}) \tilde{u}(n) .
$$

We have

$$
V_{d}(n) \sim \frac{2}{3} \frac{\sqrt{3}}{(24 n)^{3 / 4}} \exp \left(\frac{\pi}{6} \sqrt{24 n}\right) \quad \text { and } \quad \tilde{u}(n) \sim \frac{\sqrt{3}}{(24 n)^{3 / 4}} \exp \left(\frac{\pi}{6} \sqrt{24 n}\right) .
$$

It follows from Fristedt [1993, Theorem 9.1] that the smallest part of a partition into distinct parts has size $j$ with probability roughly equal to $1 / 2^{j}$. Therefore, a pair of partitions into distinct parts will have the same smallest part with probability roughly equal to $\frac{1}{3}$, which agrees with the prediction.

It would be of interest to address some of the following questions as $n \rightarrow \infty$ :

(1) What is the distribution of the center part of a (strongly) concave composition?

(2) How many parts does a typical (strongly) concave composition of $n$ have? 
(3) What is the distribution of the number of parts to the left of the center part minus the number of parts to the right of the center part?

(4) What is the distribution of the perimeter of the (strongly) concave composition of $n$ ?

Some of these questions can be answered by modeling a concave composition as the convolution of two random partitions (discussed above), while others can be treated via modular techniques of Bringmann, Mahlburg and the second author [Bringmann et al. 2012].

It is convenient to introduce a two-variable generating function. Let $R-L$ be the rank of the concave composition. This quantity measures the position of the central part. Let $V(m, n)$ and $V_{d}(m, n)$ be the numbers of concave compositions and strongly concave compositions, respectively, of $n$ with rank equal to $m$. Standard techniques give

$$
\begin{aligned}
v(x, q) & :=\sum_{\substack{n \geq 0 \\
m \in \mathbb{Z}}} V(m, n) x^{m} q^{n}=\sum_{n=0}^{\infty} \frac{q^{n}}{\left(x q^{n+1} ; q\right)_{\infty}\left(x^{-1} q^{n+1} ; q\right)_{\infty}} \\
v_{d}(x, q) & :=\sum_{\substack{n \geq 0 \\
m \in \mathbb{Z}}} V_{d}(m, n) x^{m} q^{n}=\sum_{n=0}^{\infty} q^{n}\left(-x q^{n+1} ; q\right)_{\infty}\left(-x^{-1} q^{n+1} ; q\right)_{\infty} .
\end{aligned}
$$

The following identities are deduced in a similar manner to Theorem 1 of [Andrews 2013].

Theorem 1.6. In the notation above,

$$
\begin{aligned}
& v_{d}(x, q)=-x \sum_{n=0}^{\infty}(-1)^{n} x^{2 n} q^{\frac{n(n+1)}{2}}+(x)_{\infty}\left(x^{-1} q\right)_{\infty} \sum_{n=0}^{\infty}\left(\frac{12}{n}\right) x^{\frac{n-1}{2}} q^{\frac{n^{2}-1}{24}}, \\
& q v(x, q)=-\frac{1}{(x)_{\infty}\left(x^{-1}\right)_{\infty}} \sum_{n=0}^{\infty} \frac{q^{n^{2}}}{(x q)_{n}\left(x^{-1} q\right)_{n}} \\
& +\frac{(1-x)}{(q)_{\infty}(x)_{\infty}\left(x^{-1}\right)_{\infty}} \sum_{n \in \mathbb{Z}} \frac{(-1)^{n} q^{\left(n^{2}+n\right) / 2} x^{-n}}{1-x q^{n}} .
\end{aligned}
$$

One should compare these generating functions to the rank of a partition studied in [Bringmann et al. 2012]. As is the case in [Bringmann et al. 2012] the moments of this rank statistic can be calculated precisely. We expect this statistic to be asymptotically asymptotically distributed; see [Diaconis et al. 2013] for a similar result in the case of the partition rank.

In Section 2 we recall some basic facts about holomorphic and nonholomorphic modular forms. In Section 3 we prove Theorem 1.1. In Section 4 we prove Theorem 1.2. In Section 5 we give the first proof of Theorem 1.3, which proceeds 
via elliptic properties of Appell sums. In Section 6 we use $q$-series manipulations to prove Theorem 1.3. In Section 7 we prove Theorem 1.3 via "modular methods". Section 8 contains a calculation of the holomorphic projection operation which relates to Theorem 1.3. In Sections 9 and 10 we prove Theorems 1.4 and 1.5. Both use the circle method, modular properties of the generating functions of interest, and asymptotic analysis. Finally, in Section 11 we discuss the analog of Theorem 1.3 for the function $v_{1}(q)$.

Throughout the remainder of the text we let $q:=e^{2 \pi i \tau}$, with $\tau=x+i y$ for $x, y \in \mathbb{R}$ and $y>0$. We let $z \in \mathbb{R}^{+}, h, k \in \mathbb{N}_{0}, 0 \leq h \leq k$ with $(h, k)=1$. Moreover, we denote by $[a]_{b}$ the inverse of $a$ modulo $b$.

\section{Holomorphic modular forms and harmonic weak Maass forms}

In this section we define and give some basic properties of harmonic weak Maass forms. Before turning to nonholomorphic modular forms we describe the classic holomorphic modular forms of half integral weight.

We follow Shimura [1973], see also [Ono 2004], by setting

$$
\left(\frac{a}{b}\right)=\eta\left(\frac{a}{|b|}\right)
$$

where $\eta=-1$ if $a, b,<0$ and $\eta=1$ if $a>0$ or $b>0$. For an odd integer $m$, we put $\epsilon_{m}=1$ if $m \equiv 3(\bmod 4)$ and $\epsilon_{m}=i$ if $m \equiv 3(\bmod 4)$. For all $\gamma=\left(\begin{array}{ll}a & b \\ c & d\end{array}\right) \in \Gamma_{0}(4)$, let

$$
j(\gamma, \tau):=\left(\frac{c}{d}\right) \epsilon_{d}^{-1}(c \tau+d)^{1 / 2} .
$$

The Dedekind $\eta$-function is defined by $\eta(\tau):=q^{1 / 24} \prod_{n=1}^{\infty}\left(1-q^{n}\right)$ and define $\chi\left(h,[-h]_{k}, k\right)$ to be the multiplier so that

$$
\eta\left(\frac{1}{k}(h+i z)\right)=\sqrt{\frac{i}{z}} \chi\left(h,[-h]_{k}, k\right) \eta\left(\frac{1}{k}\left([-h]_{k}+\frac{i}{z}\right)\right) .
$$

By [Ono 2004, Theorem 1.60], for instance,

$$
\eta(8 \tau)^{3}=\sum_{n \in \mathbb{Z}}(-1)^{n}(2 n+1) e^{2 \pi i(2 n+1)^{2} \tau}=\sum_{n=1}^{\infty}\left(\frac{-4}{n}\right) n q^{n^{2}} .
$$

The next result follows from Theorem 1.44 of [Ono 2004].

Lemma 2.1. $\eta(8 \tau)^{3}$ is a weight $-\frac{3}{2}$ modular form on $\Gamma_{0}(64)$ with trivial Nebentypus.

Before discussing harmonic weak Maass forms we introduce the quasimodular form

$$
E_{2}(\tau):=1-24 \sum_{n=1}^{\infty} \sigma_{1}(n) q^{n}
$$


where $\sigma_{1}(n):=\sum_{d \mid n} d$. In particular it satisfies $E_{2}(\tau+1)=E_{2}(\tau)$ and

$$
E_{2}(\tau)=\tau^{-2} E_{2}\left(-\frac{1}{\tau}\right)-\frac{6}{\pi i \tau} .
$$

Therefore, the completed form of $E_{2}$ defined by

$$
\widehat{E}_{2}(\tau):=E_{2}(\tau)-\frac{3}{\pi y}
$$

transforms as a modular form of weight 2 for $\mathrm{SL}_{2}(\mathbb{Z})$.

We turn to harmonic weak Maass forms. Define the weight $k$ hyperbolic Laplacian by

$$
\Delta_{k}:=-y^{2}\left(\frac{\partial^{2}}{\partial x^{2}}+\frac{\partial^{2}}{\partial y^{2}}\right)+i k y\left(\frac{\partial}{\partial x}+i \frac{\partial}{\partial y}\right) .
$$

Definition 2.2. Suppose that $k \in \frac{1}{2} \mathbb{Z}, N$ is a positive integer, and that $\psi$ is a Dirichlet character with modulus $4 N$. A harmonic weak Maass form of weight $k$ on $\Gamma_{0}(4 N)$ with Nebentypus character $\psi$ is a smooth function $f: \mathbb{H} \rightarrow \mathbb{C}$ satisfying the following:

(1) For all $A=\left(\begin{array}{ll}a & b \\ c & d\end{array}\right) \in \Gamma_{0}(4 N)$ and all $\tau \in \mathbb{H}$, we have

$$
f(A \tau)=\psi(d) j(A, \tau)^{2 k} f(\tau) .
$$

(2) We have $\Delta_{k} f=0$.

(3) The function $f$ has at most linear exponential growth at all the cusps of $\mathbb{H} / \Gamma_{0}(N)$.

In [Zwegers 2002] a general class of harmonic weak Maass forms was constructed by "completing" certain Appell sums. The Appell sum is defined for $u, v \in \mathbb{C} \backslash$ $(\mathbb{Z}+\mathbb{Z} \tau)$ by

$$
\mu(u, v ; \tau):=\frac{e^{\pi i u}}{\vartheta(v ; \tau)} \sum_{n \in \mathbb{Z}} \frac{(-1)^{n} e^{2 \pi i n v} q^{n(n+1) / 2}}{1-e^{2 \pi i u} q^{n}}
$$

where

$$
\vartheta(v ; \tau):=\sum_{v \in \frac{1}{2}+\mathbb{Z}} e^{\pi i v^{2} \tau+2 \pi i v\left(v+\frac{1}{2}\right)}
$$

is the Jacobi theta function. The Jacobi theta function satisfies the triple product identity

$$
\vartheta(v ; \tau)=-i q^{1 / 8} \zeta^{-1 / 2} \prod_{n=1}^{\infty}\left(1-q^{n}\right)\left(1-\zeta q^{n-1}\right)\left(1-\zeta^{-1} q^{n}\right)
$$

with $\zeta=e^{2 \pi i v}$, and the transformation 


$$
\vartheta\left(-i v z ; \frac{1}{k}(h+i z)\right)=\chi^{3}\left(h,[-h]_{k}, k\right) \sqrt{\frac{i}{z}} e^{\pi k z v^{2}} \vartheta\left(v, \frac{1}{k}\left([-h]_{k}+\frac{i}{z}\right)\right) .
$$

The nonholomorphic correction term of the Appell sum requires the definition

$$
R(u ; \tau):=\sum_{\nu \in \mathbb{Z}+\frac{1}{2}}(-1)^{\nu-\frac{1}{2}}\left\{\operatorname{sgn}(v)-E\left(\left(v+\frac{\operatorname{Im}(u)}{\operatorname{Im}(\tau)}\right) \sqrt{2 \operatorname{Im}(\tau)}\right)\right\} e^{-2 \pi i v u} e^{-\pi i v^{2} \tau},
$$

with $E(x)$ defined by

$$
E(x):=2 \int_{0}^{x} e^{-\pi u^{2}} d u=\operatorname{sgn}(x)\left(1-\beta\left(x^{2}\right)\right),
$$

where for positive real $x$ we let $\beta(x):=\int_{x}^{\infty} u^{-1 / 2} e^{-\pi u} d u$. We have the following useful properties of $R$.

Proposition 2.3 [Zwegers 2002, Propositions 1.9 and 1.10]. If $u \in \mathbb{C}$ and $\operatorname{Im}(\tau)>0$, then

(1) $R(u+1 ; \tau)=R(-u ; \tau)=-R(u ; \tau)$

(2) $R(u ; \tau+1)=e^{-\pi i / 4} R(u ; \tau)$,

(3) $R(u ; \tau)=-\sqrt{\frac{i}{\tau}} e^{i \pi u^{2} / \tau}\left(R\left(\frac{u}{\tau} ;-\frac{1}{\tau}\right)-H\left(\frac{u}{\tau} ;-\frac{1}{\tau}\right)\right)$,

where the Mordell integral is defined by

$$
H(u ; z):=\int_{-\infty}^{\infty} \frac{e^{\pi i z x^{2}-2 \pi x u}}{\cosh (\pi x)} d x .
$$

Moreover, we need the following "dissection" property of $R$.

Proposition 2.4 [Bringmann and Folsom 2013, Proposition 2.3]. For $n \in \mathbb{N}$, we have

$$
\begin{aligned}
& R\left(u ; \frac{z}{n}\right) \\
& \quad=\sum_{\ell=0}^{n-1} q^{-\frac{1}{2 n}\left(\ell-\frac{n-1}{2}\right)^{2}} e^{-2 \pi i\left(\ell-\frac{n-1}{2}\right)\left(u+\frac{1}{2}\right)} R\left(n u+\left(\ell-\frac{n-1}{2}\right) z+\frac{n-1}{2} ; n z\right) .
\end{aligned}
$$

The completion of $\mu$ is defined by

$$
\widehat{\mu}(u, v ; \tau):=\mu(u, v ; \tau)+\frac{i}{2} R(u-v ; \tau) .
$$

This function satisfies the following elliptic and modular transformation laws.

Theorem 2.5 [Zwegers 2002, Theorem 1.11]. Assume all of the notation and hypotheses from above. If $k, \ell, m, n \in \mathbb{Z}$, then we have

$\widehat{\mu}(u+k \tau+\ell, v+m \tau+n ; \tau)=(-1)^{k+\ell+m+n} e^{\pi i \tau(k-m)^{2}+2 \pi i(k-m)(u-v)} \widehat{\mu}(u, v ; \tau)$ 
and

$\widehat{\mu}\left(-i u z,-i v z ; \frac{h+i z}{k}\right)=\chi^{-3}\left(h,[-h]_{k}, k\right) \sqrt{\frac{i}{z}} e^{-\pi k z(u-v)^{2}} \hat{\mu}\left(u, v ; \frac{1}{k}\left([-h]_{k}+\frac{i}{z}\right)\right)$.

Finally, we have a result that is useful in determining the shadow of a mock theta function.

Theorem 2.6 [Zwegers 2002, Theorem 1.16]. For $a \in\left(-\frac{1}{2}, \frac{1}{2}\right)$ and $b \in \mathbb{R}$ we have

$$
\int_{-\bar{\tau}}^{i \infty} \frac{g_{a+\frac{1}{2}, b+\frac{1}{2}}(z)}{\sqrt{-i(\tau+z)}} d z=-e^{-\pi i a^{2} \tau+2 \pi i a\left(b+\frac{1}{2}\right)} R(a \tau-b ; \tau)
$$

where $g_{a, b}(z):=\sum_{v \in a+\mathbb{Z}} v e^{\pi i v^{2} z+2 \pi i v b}$.

\section{Proof of Theorem 1.1}

In this section we use the Jacobi properties of the Appell sums

$$
\begin{gathered}
A(u, v ; \tau):=e^{\pi i u} \sum_{n \in \mathbb{Z}} \frac{(-1)^{n} e^{2 \pi i n v} q^{\left(n^{2}+n\right) / 2}}{1-e^{2 \pi i u} q^{n}}, \\
A_{3}(u ; \tau):=e^{\pi i u} \sum_{n \in \mathbb{Z}} \frac{(-1)^{n} q^{\left(3 n^{2}+n\right) / 2}}{1-e^{2 \pi i u} q^{n}},
\end{gathered}
$$

to deduce Theorem 1.1. The function $A_{3}(u ; \tau)$ was studied by Bringmann [2008].

Direct computation gives

$$
\begin{aligned}
& f_{2}(\tau):=\sum_{n \neq 0} \frac{(-1)^{n+1} q^{\frac{3 n(n+1)}{2}}}{\left(1-q^{n}\right)^{2}}=\left.\left(\frac{1}{2 \pi i} \frac{\partial}{\partial u}\left(e^{-\pi i u} A_{3}(u, \tau)-\frac{1}{1-e^{2 \pi i u}}\right)\right)\right|_{u=0}, \\
& f_{1}(\tau):=\sum_{n \neq 0} \frac{(-1)^{n+1} n q^{\frac{n(n+1)}{2}}}{1-q^{n}}=-\left.\frac{1}{2 \pi i} \frac{\partial}{\partial v}\right|_{u=v=0} A(u, v ; \tau) .
\end{aligned}
$$

Thus the modular properties of the Appell sums will dictate the modular properties of our functions $v_{1}(q)$ and $v_{2}(q)$. In analogy to (2-12) the completed forms of these sums are

$$
\begin{aligned}
\widehat{A}(u, v ; \tau) & :=A(u, v ; \tau)+\frac{i}{2} \vartheta(v ; \tau) R(u-v ; \tau), \\
\widehat{A}_{3}(u ; \tau) & :=A_{3}(u ; \tau)+\frac{1}{2} \eta(\tau) q^{-\frac{1}{6}}\left(e^{2 \pi i u} R(3 u-\tau ; 3 \tau)-e^{-2 \pi i u} R(3 u+\tau ; 3 \tau)\right) .
\end{aligned}
$$

Using the transformation properties of $\mu$ found in Chapter 1 of [Zwegers 2002], we have the following modular properties of $\hat{A}$. For $\hat{A}_{3}$ we will use Theorem 3.1 of [Bringmann 2008]. 
Proposition 3.1. For all $\left(\begin{array}{ll}a & b \\ c & d\end{array}\right) \in \mathrm{SL}_{2}(\mathbb{Z})$ we have

$$
\widehat{A}\left(\frac{u}{c \tau+d}, \frac{v}{c \tau+d} ; \frac{a \tau+b}{c \tau+d}\right)=(c \tau+d) e^{\pi i \frac{c}{c \tau+d}\left(2 u v-v^{2}\right)} \widehat{A}(u, v ; \tau) .
$$

We then have the following theorem which together with the transformation properties of $\widehat{E}_{2}(\tau)$ and (1-2) yields Theorem 1.1 .

Theorem 3.2. With $\tau=x+i y$ for $x, y \in \mathbb{R}$ and $y>0$ we let

$$
\begin{aligned}
& \widehat{f_{1}}(\tau)=f_{1}(\tau)-\frac{1}{4 \pi y}-\frac{i}{2} \eta(\tau)^{3} \int_{-\bar{\tau}}^{i \infty} \frac{\eta(z)^{3}}{(-i(z+\tau))^{\frac{1}{2}}} d z, \\
& \hat{f}_{2}(\tau)=f_{2}(\tau)-\frac{1}{24}+\frac{3}{8 \pi y}+\frac{\sqrt{3}}{2 \pi i} \eta(\tau) \int_{-\bar{\tau}}^{i \infty} \frac{\eta(z)}{(-i(z+\tau))^{\frac{3}{2}}} d z .
\end{aligned}
$$

Both $\hat{f}_{1}$ and $\hat{f}_{2}$ transform as a weight 2 modular form for all of $\mathrm{SL}_{2}(\mathbb{Z})$ with trivial Nebentypus.

Proof. We establish the claim for $f_{1}$ first. We have

$$
\begin{aligned}
\left.\frac{1}{2 \pi i} \frac{\partial}{\partial v}\right|_{u=v=0} \widehat{A}(u, v ; \tau) & =-f_{1}(\tau)+\frac{1}{4 \pi} \vartheta^{\prime}(0) R(0 ; \tau) \\
& =-f_{1}(\tau)+\frac{i}{2} \eta(\tau)^{3} \int_{-\bar{\tau}}^{i \infty} \frac{\eta(z)^{3}}{(-i(z+\tau))^{\frac{1}{2}}} d z \\
& =-\widehat{f}_{1}(\tau)-\frac{1}{4 \pi y}
\end{aligned}
$$

where we have used Theorem 2.6 in the second equality and the fact that $\vartheta(0)=0$.

Additionally, from Proposition 3.1 we have (by taking $(1 / 2 \pi i) \partial /\left.\partial v\right|_{u=v=0}$ on both sides)

$$
\begin{aligned}
& \frac{1}{(c \tau+d)}\left(-\widehat{f}_{1}\left(\frac{a \tau+b}{c \tau+d}\right)-\frac{1}{4 \pi} \cdot \frac{1}{\operatorname{Im}\left(\frac{a \tau+b}{c \tau+d}\right)}\right) \\
& \quad=(c \tau+d)\left(-\widehat{f}_{1}(\tau)-\frac{1}{4 \pi y}\right)+c \lim _{u, v \rightarrow 0} u e^{\pi i \frac{c}{c \tau+d}(2 u v-u)^{2}} \widehat{A}(u, v ; \tau)
\end{aligned}
$$

Using

$$
\lim _{u \rightarrow 0} u \widehat{A}(u, v ; \tau)=-\frac{1}{2 \pi i} \quad \text { or } \quad \lim _{v \rightarrow 0} \widehat{A}(u, v ; \tau)=-\frac{1}{2 \pi i} \frac{\vartheta^{\prime}(0)}{\vartheta(u)}
$$

we find that

$$
\lim _{u, v \rightarrow 0} u e^{\pi i \frac{c}{c \tau+d}\left(2 u v-u^{2}\right)} \widehat{A}(u, v ; \tau)=-\frac{1}{2 \pi i}
$$


Additionally, since

$$
\operatorname{Im}\left(\frac{a \tau+b}{c \tau+d}\right)=\frac{y}{|c \tau+d|^{2}}
$$

we have

$$
\begin{aligned}
(c \tau+d) \widehat{f}_{1}(\tau)-\frac{1}{(c \tau+d)} \widehat{f}_{1}\left(\frac{a \tau+b}{c \tau+d}\right) & =\frac{1}{c \tau+d} \frac{1}{4 \pi \operatorname{Im}\left(\frac{a \tau+b}{c \tau+d}\right)}-\frac{c \tau+d}{4 \pi y}-\frac{c}{2 \pi i} \\
& =\frac{c \bar{\tau}+d}{4 \pi y}-\frac{c \tau+d}{4 \pi y}-\frac{c}{2 \pi i}=0
\end{aligned}
$$

which gives the result for $\widehat{f}_{1}$.

We define

$$
\widetilde{A}_{3}(u ; \tau)=e^{-\pi i u} A_{3}(u, \tau)-\frac{1}{1-e^{2 \pi i u}} .
$$

By Theorem 3.1 of [Bringmann 2008] we have

$$
\begin{aligned}
\widetilde{A}_{3}(u, \tau)= & \frac{1}{1-e^{2 \pi i u}}-\frac{i e^{\frac{3 \pi u^{2}}{\tau}}-\pi i u-\frac{\pi u}{\tau}}{\tau\left(1-e^{-\frac{2 \pi u}{\tau}}\right)}+\frac{i}{\tau} e^{\frac{3 \pi u^{2}}{\tau}-\pi i u-\frac{\pi u}{\tau}} \widetilde{A}_{3}\left(\frac{i u}{\tau},-\frac{1}{\tau}\right) \\
& -(-i \tau)^{-\frac{1}{2}} \eta\left(-\frac{1}{\tau}\right) \int_{\mathbb{R}} e^{3 \pi i \tau x^{2}}\left(\sum_{ \pm} \frac{e^{ \pm \frac{\pi i}{6} \mp \pi i \tau x}}{1-e^{2 \pi i u} e^{ \pm \frac{\pi i}{3} \mp 2 \pi i \tau x}}\right) d x
\end{aligned}
$$

Remark. This may also be derived by using

$$
\begin{gathered}
e^{3 \pi i u / 2} \sum_{n \in \mathbb{Z}} \frac{(-1)^{n} q^{3 n(n+1) / 2} e^{2 \pi i n v}}{1-e^{2 \pi i u} q^{n}} \\
=\sum_{k=0}^{2} e^{2 \pi i k u} \vartheta(v+k \tau+1 ; 3 \tau) \mu(3 u, v+k \tau+1 ; 3 \tau)
\end{gathered}
$$

and the results of Chapter 1 of [Zwegers 2002].

Lemma 4.2 of [Bringmann 2008] gives

$$
\begin{aligned}
\left.\frac{1}{2 \pi i} \frac{\partial}{\partial u}\right|_{u=0} \int_{\mathbb{R}} e^{3 \pi i \tau x^{2}}\left(\sum_{ \pm} \frac{e^{ \pm \frac{\pi i}{6} \mp \pi i \tau x}}{1-e^{2 \pi i u} e^{ \pm \frac{\pi i}{3} \mp 2 \pi i \tau x}}\right) d x & \\
& =\frac{\sqrt{3}(-i \tau)^{2}}{2 \pi} \int_{0}^{\infty} \frac{\eta(i w)}{(-i(i w-i \tau))^{\frac{3}{2}}} d w .
\end{aligned}
$$


Using the transformation $\eta(-1 / \tau)=\sqrt{-i \tau} \eta(\tau)$ and applying $(1 / 2 \pi i) \partial /\left.\partial u\right|_{u=0}$ to (3-2), we have

$$
\begin{aligned}
& f_{2}(\tau) \\
& =\left.\frac{1}{2 \pi i} \frac{\partial}{\partial u}\left(\frac{1}{1-e^{2 \pi i u}}+\frac{e^{\frac{3 \pi i u^{2}}{\tau}-\pi i u-\frac{\pi i u}{\tau}}}{\tau\left(1-e^{-\frac{2 \pi i u}{\tau}}\right)}+\frac{1}{\tau} e^{\frac{3 \pi i u^{2}}{\tau}-\pi i u-\frac{\pi i u}{\tau}} \tilde{A}_{3}\left(\frac{u}{\tau},-\frac{1}{\tau}\right)\right)\right|_{u=0} \\
& +\tau^{2} \eta(\tau) \frac{\sqrt{3}}{2 \pi} \int_{0}^{\infty} \frac{\eta(i w)}{(-i(i w-i \tau))^{\frac{3}{2}}} d w \\
& =\left.\frac{1}{2 \pi i} \frac{\partial}{\partial u}\left(\frac{1}{1-e^{2 \pi i u}}+\frac{e^{\frac{3 \pi i u^{2}}{\tau}-\pi i u-\frac{\pi u}{\tau}}}{\tau\left(1-e^{-\frac{2 \pi i u}{\tau}}\right)}\right)\right|_{u=0}+\tau^{-2} f_{2}\left(-\frac{1}{\tau}\right) \\
& +\tau^{2} \eta(\tau) \frac{\sqrt{3}}{2 \pi} \int_{0}^{\infty} \frac{\eta(i w)}{(-i(i w-i \tau))^{\frac{3}{2}}} d w \\
& =\left(\frac{1}{24}-\frac{1}{24 \tau^{2}}+\frac{3}{4 \pi i \tau}\right)+\tau^{-2} f_{2}\left(-\frac{1}{\tau}\right) \\
& +\tau^{2} \eta(\tau) \frac{\sqrt{3}}{2 \pi} \int_{0}^{\infty} \frac{\eta(i w)}{(-i(i w-i \tau))^{\frac{3}{2}}} d w,
\end{aligned}
$$

where we have used

$$
\sum_{n \neq 0} \frac{(-1)^{n} q^{\frac{3 n^{2}+n}{2}}}{1-q^{n}}=0
$$

Next define

$$
\mathscr{S}(\tau):=\frac{\sqrt{3}}{2 \pi i} \eta(\tau) \int_{-\bar{\tau}}^{i \infty} \frac{\eta(w)}{(-i(w+\tau))^{\frac{3}{2}}} d w .
$$

Then the modular transformation of $\eta(\tau)$ implies that

$$
\mathscr{\varphi}(\tau)=\tau^{2} \varphi\left(-\frac{1}{\tau}\right)-\tau^{2} \frac{\sqrt{3}}{2 \pi} \int_{0}^{\infty} \frac{\eta(i w)}{(-i(i w-i \tau))^{\frac{3}{2}}} d w .
$$

Combining this with (3-3) gives $\hat{f}_{2}(\tau)=\tau^{-2} \hat{f}_{2}(-1 / \tau)$. Using $\hat{f}_{2}(\tau+1)=\hat{f}_{2}(\tau)$, we obtain the result.

Proof of Theorem 1.1. By (1-2) and

$$
\sum_{n=1}^{\infty} \frac{q^{n}}{\left(1-q^{n}\right)^{2}}=\sum_{n=1}^{\infty} \frac{n q^{n}}{1-q^{n}}=\sum_{n \geq 1} \sigma_{1}(n) q^{n}=\frac{1-E_{2}(\tau)}{24}
$$

we have $q(q)_{\infty}^{3} v(q)=f_{1}(\tau)+f_{2}(\tau)+\frac{1}{24}\left(E_{2}(\tau)-1\right)$. The result now follows from Theorem 3.2 and (2-5). 


\section{Proof of Theorem 1.2}

In this section we give the following more precise version of Theorem 1.2.

Theorem 4.1. With $q=e^{2 \pi i \tau}$ we have

$$
q^{-1}\left(q^{24} ; q^{24}\right)_{\infty}^{2} v_{1}\left(q^{24}\right)+\frac{i}{4 \sqrt{2} \pi} \int_{-\bar{\tau}}^{i \infty} \frac{\eta(24 z)}{(-i(\tau+z))^{\frac{3}{2}}} d z
$$

is a harmonic weak Maass form of weight $\frac{3}{2}$ on $\Gamma_{0}(576)$ with Nebentypus $\left(\frac{12}{-}\right)$. Also,

$$
q^{-1} v_{2}\left(q^{8}\right)-i \sqrt{2} \int_{-\bar{\tau}}^{i \infty} \frac{\eta(8 z)^{3}}{\sqrt{-i(z+\tau)}} d z
$$

is a harmonic weak Maass form of weight $\frac{1}{2}$ on $\Gamma_{0}(64)$ with trivial Nebentypus. Finally, let

$$
E_{2}^{\mathrm{odd}}(\tau):=1+24 \sum_{n>0}\left(\sum_{\substack{d \mid n \\ d o d d}} d\right) q^{n}=2 E_{2}(2 \tau)-E_{2}(\tau) .
$$

Then

$$
q^{-1} v_{3}\left(q^{8}\right)=\frac{E_{2}^{\mathrm{odd}}(8 \tau)}{6 \eta(8 \tau)^{3}}
$$

is a weight $-\frac{1}{2}$ weakly holomorphic modular form on $\Gamma_{0}(64)$ with trivial Nebentypus.

The modular properties of $v_{1}(q)$ and $v_{3}(q)$ are straightforward or follow from known results. Similar to (3-4) we have

$$
\sum_{n=1}^{\infty} \frac{q^{n}}{\left(1+q^{n}\right)^{2}}=\sum_{n=1}^{\infty} \frac{(-1)^{n+1} n q^{n}}{1-q^{n}}=-\sum_{n \geq 1}\left(\sum_{d \mid n}(-1)^{d} d\right) q^{n} .
$$

Hence

$$
\sum_{n=1}^{\infty} q^{n}\left(\frac{1}{\left(1+q^{n}\right)^{2}}+\frac{1}{\left(1-q^{n}\right)^{2}}\right)=\frac{E_{2}^{\mathrm{odd}}(\tau)-1}{12}
$$

The claim for $v_{2}(q)$ follows from Lemma 2.1 and the fact that $E^{\text {odd }}(\tau)$ is a holomorphic modular form of weight 2 on $\Gamma_{0}(4)$. The proof of the claims about $v_{1}(q)$ follows from (3-4) and the following theorem of Bringmann [2008].

Theorem 4.2 [Bringmann 2008, Theorem 1.1]. Let $q=e^{2 \pi i \tau}$ and

$$
\mathscr{R}(\tau):=\frac{q^{-1}}{\left(q^{24} ; q^{24}\right)_{\infty}} \sum_{n \neq 0} \frac{(-1)^{n+1} q^{36 n(n+1)}}{\left(1-q^{24 n}\right)^{2}} .
$$


Then

$$
M(\tau):=\mathscr{R}(\tau)-\frac{i}{4 \sqrt{2} \pi} \int_{-\bar{\tau}}^{i \infty} \frac{\eta(24 z)}{(-i(\tau+z))^{\frac{3}{2}}} d z-\frac{1}{24 \eta(24 \tau)}+\frac{E_{2}(24 \tau)}{8 \eta(24 \tau)}
$$

is a harmonic weak Maass form of weight $\frac{3}{2}$ on $\Gamma_{0}(576)$ with Nebentypus $\left(\frac{12}{.}\right)$.

The modularity of $v_{2}(q)$ follows easily from the identity of Theorem 1.3 and the results of Chapter 1 of [Zwegers 2002], namely, the following theorem.

Theorem 4.3. With $q=e^{2 \pi i \tau}$ we have $\widetilde{F}(q)=-2 i q^{1 / 8} \mu\left(\frac{1}{2}, \frac{1}{2} ; \tau\right)$ is a mock theta function with shadow proportional to $\eta^{3}(z)$. More precisely,

$$
q^{-1} \tilde{F}\left(q^{8}\right)+2 i \sqrt{2} \int_{-\bar{\tau}}^{i \infty} \frac{\eta(8 z)^{3}}{\sqrt{-i(z+\tau)}} d z
$$

is a harmonic weak Maass form of weight $\frac{1}{2}$ on $\Gamma_{0}(64)$ with trivial Nebentypus.

Proof. Since this function is written in terms of $\mu$ the result follows from Chapter 1 of [Zwegers 2002]. In particular, to compute the shadow we use Theorem 2.6 and Lemma 2.1. For additional details, see [Eguchi and Hikami 2009; Rhoades 2012].

We have used Theorem 1.3 to establish the modularity of $v_{2}(q)$. In the next three sections we will give different proofs of this theorem. Each proof uses different techniques and highlights different aspects of the Appell sums appearing in Theorem 1.3.

\section{Elliptic proof of Theorem 1.3}

In this section we prove the identity of Theorem 1.3 via a transformation property of $\mu(u, v ; \tau)$ with respect to the elliptic variables $u$ and $v$.

Proposition 5.1 [Zwegers 2002, Proposition 1.4(7)]. For $u, v, u+z, v+z \notin \mathbb{Z} \tau+\mathbb{Z}$, we have

$$
\mu(u+z, v+z ; \tau)-\mu(u, v ; \tau)=\frac{1}{2 \pi i} \frac{\vartheta^{\prime}(0) \vartheta(u+v+z) \vartheta(z)}{\vartheta(u) \vartheta(v) \vartheta(u+z) \vartheta(v+z)},
$$

where we write $\vartheta(u)=\vartheta(u ; \tau)$ when $\tau$ is understood.

Let

$$
F_{2 k}(\tau):=\sum_{n=1}^{\infty} \sum_{d \mid n}(-1)^{d} d^{2 k-1} q^{n} \quad \text { and } \quad \Phi_{2 k}(\tau):=\sum_{n=1}^{\infty} \sum_{d \mid n} d^{2 k-1} q^{n} .
$$

We will need the following lemma of [Rhoades 2013]. 
Lemma 5.2. With $Z=2 \pi i u$ we have

$$
\begin{gathered}
\vartheta(u ; \tau)=-2 \sin (\pi u) \eta^{3}(\tau) \exp \left(-2 \sum_{\substack{\ell \text { even } \\
\ell>0}} \frac{Z^{\ell}}{\ell !} \Phi_{\ell}(\tau)\right), \\
\vartheta\left(u+\frac{1}{2} ; \tau\right)=-2 \cos (\pi u) \frac{\eta(2 \tau)^{2}}{\eta(\tau)} \exp \left(-2 \sum_{\substack{\ell>0 \\
\ell \text { even }}} \frac{Z^{\ell}}{\ell !} F_{\ell}(\tau)\right) .
\end{gathered}
$$

With this lemma we turn to the proof of Theorem 1.3 using Proposition 5.1.

"Elliptic" proof of Theorem 1.3. Throughout this proof let $x=e^{2 \pi i u}$. From Proposition 5.1 and using $\vartheta^{\prime}(0)=-2 \pi \eta(\tau)^{3}$, we have

$$
\vartheta(u) \vartheta(-u) \mu\left(u+\frac{1}{2},-u+\frac{1}{2} ; \tau\right)-\vartheta(u) \vartheta(-u) \mu(u,-u ; \tau)=\frac{i \eta^{3}(\tau) \vartheta\left(\frac{1}{2}\right)^{2}}{\vartheta\left(u+\frac{1}{2}\right)^{2}} .
$$

Taylor expanding each of the three terms in this identity around $u=0$ by applying Lemma 5.2, we have

$$
\begin{gathered}
\vartheta(u) \vartheta(-u) \mu\left(u+\frac{1}{2},-u+\frac{1}{2} ; \tau\right)=-4 i \pi^{2}\left(\frac{\eta(\tau)^{6}}{\vartheta\left(\frac{1}{2}\right)} \sum_{n \in \mathbb{Z}} \frac{q^{\frac{n^{2}+n}{2}}}{1+q^{n}}\right) u^{2}+O\left(u^{4}\right), \\
\frac{i \eta^{3}(\tau) \vartheta\left(\frac{1}{2}\right)}{\vartheta\left(u+\frac{1}{2}\right)^{2}}=i \eta(\tau)^{3}+4 i \pi^{2}\left(\frac{1}{4}-2 F_{2}(\tau)\right) u^{2}+O\left(u^{4}\right) .
\end{gathered}
$$

The remaining term is more interesting. From the definition of $\mu(u,-u ; \tau)$, we have

$$
\vartheta(u) \vartheta(-u) \mu(u,-u ; \tau)=\frac{i \vartheta(u)}{2 \sin (\pi u)}+\vartheta(u ; \tau) e^{\pi i u} \sum_{n \neq 0} \frac{(-1)^{n} x^{-n} q^{\left(n^{2}+n\right) / 2}}{1-x q^{n}} .
$$

Since

$$
\sum_{n \neq 0} \frac{(-1)^{n} q^{\left(n^{2}+n\right) / 2}}{1-q^{n}}=0
$$

we have

$$
\begin{aligned}
& \sum_{n \neq 0} \frac{(-1)^{n} x^{-n} q^{\left(n^{2}+n\right) / 2}}{1-x q^{n}} \\
& \quad=2 \pi i u\left(\sum_{n \neq 0} \frac{(-1)^{n+1} n q^{\left(n^{2}+n\right) / 2}}{1-q^{n}}+\sum_{n \neq 0} \frac{(-1)^{n} q^{\left(n^{2}+n\right) / 2}}{\left(1-q^{n}\right)^{2}}\right)+O\left(u^{2}\right) .
\end{aligned}
$$

Again applying Lemma 5.2 we have 


$$
\begin{aligned}
& -\vartheta(u) \vartheta(-u) \mu(u,-u ; \tau) \\
& =i \eta^{3}(\tau)-4 i \pi^{2} \eta^{3}(\tau) \\
& \times\left(\sum_{n \neq 0} \frac{(-1)^{n} n q^{\left(n^{2}+n\right) / 2}}{1-q^{n}}-\sum_{n \neq 0} \frac{(-1)^{n} q^{\left(n^{2}+n\right) / 2}}{\left(1-q^{n}\right)^{2}}-\Phi_{1}(\tau)\right) u^{2}+O\left(u^{3}\right) .
\end{aligned}
$$

Using (5-2), (5-3), (5-4), and (5-5) to compare the $u^{2}$ coefficient of the Taylor expansion around $u=0$ of both sides of (5-2), we have

$$
\begin{array}{r}
\frac{1}{\vartheta\left(\frac{1}{2}\right)} \sum_{n \in \mathbb{Z}} \frac{q^{\frac{n^{2}+n}{2}}}{1+q^{n}}-\frac{1}{\eta^{3}(\tau)}\left(\sum_{n \neq 0} \frac{(-1)^{n+1} n q^{\frac{n^{2}+n}{2}}}{1-q^{n}}+\sum_{n \neq 0} \frac{(-1)^{n} q^{\frac{n^{2}+n}{2}}}{\left(1-q^{n}\right)^{2}}+\Phi_{2}(\tau)\right) \\
=\frac{1}{\eta^{3}(z)}\left(-\frac{1}{4}+2 F_{2}(\tau)\right) .
\end{array}
$$

The identity follows from $\vartheta\left(\frac{1}{2}\right)=-2 q^{1 / 8}(q)_{\infty}(-q)_{\infty}^{2}, \eta^{3}(\tau)=q^{1 / 8}(q)_{\infty}^{3}$,

$$
\sum_{n \neq 0} \frac{(-1)^{n} q^{\left(n^{2}+n\right) / 2}}{\left(1-q^{n}\right)^{2}}=-\Phi_{2}(\tau), \quad \text { and } \quad \sum_{n=1}^{\infty} \frac{q^{n}}{\left(1+q^{n}\right)^{2}}=-F_{2}(\tau) \text {. }
$$

\section{6. $q$-Series proof of Theorem 1.3}

In this section we will give a $q$-series proof of the identity in Theorem 1.3. We will divide the proof into several lemmas.

For negative values of $n$ we define $(x)_{n}=(x)_{\infty} /\left(x q^{n}\right)_{\infty}$. For simplicity we write $(a, b, c, d ; q)_{n}=(a ; q)_{n}(b ; q)_{n}(c ; q)_{n}(d ; q)_{n}$. We will use the equations

$$
\vartheta_{4}:=\sum_{n \in \mathbb{Z}}(-1)^{n} q^{n^{2}}=\frac{(q)_{\infty}}{(-q)_{\infty}}
$$

which is [Andrews 1998, (2.2.12)], and

$$
\begin{aligned}
& \sum_{n \in \mathbb{Z}} \frac{(e)_{n}(f)_{n}\left(\frac{a q}{e f}\right)^{n}}{\left(\frac{a q}{c}\right)_{n}\left(\frac{a q}{d}\right)_{n}} \\
& \quad=\frac{\left(\frac{q}{c}, \frac{q}{d}, \frac{a q}{e}, \frac{a q}{f} ; q\right)_{\infty}}{\left(a q, a^{-1} q, \frac{a q}{c d}, \frac{a q}{e f} ; q\right)_{\infty}} \sum_{n \in \mathbb{Z}} \frac{\left(1-a q^{2 n}\right)(c, d, e, f ; q)_{n}\left(\frac{q a^{3}}{c d e f}\right)^{n} q^{n^{2}}}{(1-a)\left(\frac{a q}{c}, \frac{a q}{d}, \frac{a q}{e}, \frac{a q}{f} ; q\right)_{n}},
\end{aligned}
$$

which can be found in [Bailey 1950] or page 135 of [Gasper and Rahman 2004].

\section{Lemma 6.1.}

$$
2 \sum_{n \in \mathbb{Z}} \frac{q^{\left(\begin{array}{c}
n+1 \\
2
\end{array}\right)}}{1+q^{n}}=\frac{1}{\vartheta_{4}^{2}}\left(1+\sum_{n=1}^{\infty}\left(\frac{8 q^{2 n^{2}+n}}{\left(1+q^{n}\right)^{2}}-\frac{16 n q^{2 n^{2}}\left(1-q^{n}\right)}{1+q^{n}}\right)\right) .
$$


Proof. In (6-2) set $e=c=-1, a=1$, and let $d, f \rightarrow \infty$ to obtain

$$
\begin{aligned}
2 \sum_{n \in \mathbb{Z}} & \frac{q^{n(n+1) / 2}}{1+q^{n}} \\
& =\frac{1}{\vartheta_{4}^{2}} \lim _{a \rightarrow 1} \sum_{n \in \mathbb{Z}} \frac{\left(1-a q^{2} n\right)}{1-a} \frac{(-1)_{n}^{2}}{(-a q)_{n}^{2}} a^{3} n q^{2 n^{2}} \\
& =\frac{1}{\vartheta_{4}^{2}}\left(1-\left.\sum_{n=1}^{\infty} \frac{d}{d a}\right|_{a=1}\left(\frac{\left(1-a q^{2 n}\right)(-1)_{n}^{2} a^{3 n} q^{2 n^{2}}}{(-a q)_{n}^{2}}+\frac{\left(1-a q^{-2 n}\right)(-1)_{-n}^{2} a^{-3 n} q^{2 n^{2}}}{(-a q)_{-n}^{2}}\right)\right) \\
& =\frac{1}{\vartheta_{4}^{2}}\left(1-\left.\frac{d}{d a}\right|_{a=1} \sum_{n=1}^{\infty}\left(1-a q^{2 n}\right)\left(\frac{(-1)_{n}}{(-a q)_{n}}\right)^{2} a^{3 n} q^{2 n^{2}}\right. \\
& \left.-\left.\frac{d}{d a}\right|_{a=1} \sum_{n=1}^{\infty}\left(1-a q^{-2 n}\right)\left(\frac{(a+1)(a+q) \cdots\left(a+q^{n-1}\right)}{(-q)_{n}^{2}}\right)^{2} a^{-3 n} q^{2 n^{2}+2 n}\right)
\end{aligned}
$$

where we use (6-1) and L'Hopital's rule in the second displayed equation. Continuing, we have

$$
\begin{aligned}
2 \sum_{n \in \mathbb{Z}} \frac{q^{n(n+1) / 2}}{1+q^{n}} & \frac{1}{\vartheta_{4}^{2}}\left(1+\sum_{n=1}^{\infty} \frac{4 q^{2 n^{2}+2 n}}{\left(1+q^{n}\right)^{2}}-\sum_{n=1}^{\infty}\left(1-q^{2 n}\right)(-1)_{n}^{2}\left(-2(-q)_{n}^{-3}\right) \sum_{j=1}^{n} \frac{q^{j}}{1+q^{j}}(-q)_{n} q^{2 n^{2}}\right. \\
& -\sum_{n=1}^{\infty}\left(1-q^{2 n}\right) \frac{12 n q^{2 n^{2}}}{\left(1+q^{n}\right)^{2}}+\sum_{n=1}^{\infty} \frac{4 q^{2 n^{2}}}{\left(1+q^{n}\right)^{2}} \\
& \left.-\sum_{n=1}^{\infty}\left(1-q^{-2 n}\right) q^{2 n^{2}+2 n} \frac{2(-1)_{n}^{2}}{(-q)_{n}^{2}} \sum_{j=0}^{n-1} \frac{1}{1+q^{j}}+\sum_{n=1}^{\infty}\left(1-q^{-2 n}\right) q^{2 n^{2}+2 n} \frac{12 n}{\left(1+q^{n}\right)^{2}}\right) \\
= & \frac{1}{\vartheta_{4}^{2}}\left(1+4 \sum_{n=1}^{\infty} \frac{q^{2 n^{2}+2 n}}{\left(1+q^{n}\right)^{2}}+8 \sum_{n=1}^{\infty}\left(1-q^{2 n}\right) \frac{q^{2 n^{2}}}{\left(1+q^{n}\right)^{2}} \sum_{j=1}^{n} \frac{q^{j}}{1+q^{j}}\right. \\
& -12 \sum_{n=1}^{\infty} \frac{n q^{2 n^{2}}\left(1-q^{n}\right)}{\left(1+q^{n}\right)}+4 \sum_{n=1}^{\infty} \frac{q^{2 n^{2}}}{\left(1+q^{n}\right)^{2}}+8 \sum_{n=1}^{\infty}\left(1-q^{2 n}\right) \frac{q^{2 n^{2}}}{\left(1+q^{n}\right)^{2}} \sum_{j=0}^{n-1} \frac{1}{1+q^{j}} \\
& \left.-12 \sum_{n=1}^{\infty} \frac{\left(1-q^{n}\right) n q^{2 n^{2}}}{\left(1+q^{n}\right)}\right)
\end{aligned}
$$


Noting that

$$
\sum_{j=1}^{n} \frac{q^{j}}{1+q^{j}}+\sum_{j=0}^{n-1} \frac{1}{1+q^{j}}=n+\frac{q^{n}}{1+q^{n}}-\frac{1}{2}
$$

we see that the right hand side equals

$$
\begin{gathered}
\frac{1}{\vartheta_{4}^{2}}\left(1+4 \sum_{n=1}^{\infty} \frac{\left(1+q^{2 n}\right) q^{2 n^{2}}}{\left(1+q^{n}\right)^{2}}-16 \sum_{n=1}^{\infty} \frac{n\left(1-q^{n}\right) q^{2 n^{2}}}{\left(1+q^{n}\right)}\right. \\
\left.+8 \sum_{n=1}^{\infty} \frac{\left(1-q^{n}\right) q^{2 n^{2}}}{\left(1+q^{n}\right)}\left(\frac{q^{n}}{1+q^{n}}-\frac{1}{2}\right)\right) \\
=\frac{1}{\vartheta_{4}^{2}}\left(1+4 \sum_{n=1}^{\infty} \frac{q^{2 n^{2}+n}}{\left(1+q^{n}\right)^{2}}-16 \sum_{n=1}^{\infty} \frac{n\left(1-q^{n}\right) q^{2 n^{2}}}{1+q^{n}}\right),
\end{gathered}
$$

which gives the result.

\section{Lemma 6.2.}

$\sum_{n=1}^{\infty} \frac{(-1)^{n-1} n q^{n(n+1) / 2}}{1-q^{n}}=\sum_{n=1}^{\infty} \frac{q^{n}}{\left(1-q^{n}\right)^{2}}-2 \sum_{n=1}^{\infty} \frac{n\left(1+q^{n}\right) q^{2 n^{2}}}{1-q^{n}}+\sum_{n=1}^{\infty} \frac{q^{2 n^{2}+n}}{\left(1-q^{n}\right)^{2}}$

Proof. Recall from the proof of (1.2) in [Andrews 2013] that

$$
\begin{aligned}
& \lim _{a, b \rightarrow 1} \frac{1}{(1-a)(1-b)}\left(S_{1}(a, b ; q)-\frac{1}{(a b ; q)_{\infty}}\right) \\
& =\frac{1}{(q)_{\infty}}\left(-\sum_{n=1}^{\infty} \frac{q^{n}}{\left(1-q^{n}\right)^{2}}+2 \sum_{n=1}^{\infty} \frac{(-1)^{n-1} n q^{n(n+1) / 2}}{1-q^{n}}\right)
\end{aligned}
$$

where

$$
S_{1}(a, b ; q)=\frac{\left(a^{-1} q ; q\right)_{\infty}}{(b q, q ; q)_{\infty}} \sum_{n \in \mathbb{Z}} \frac{(1-a)(-b)^{n} q^{n(n+1) / 2}}{1-a q^{n}}
$$

We now apply (6-2) with $f, d \rightarrow \infty$ and setting $a$ equal to $a b$. Then letting $e \rightarrow a$ we obtain

$$
S_{1}(a, b ; q)=\frac{\left(\frac{q}{a}, \frac{q}{b} ; q\right)_{\infty}}{\left(a b q, \frac{q}{a b}, q ; q\right)_{\infty}} \sum_{n \in \mathbb{Z}} \frac{\left(1-a b q^{2 n}\right)}{(1-a b)} \frac{(a, b ; q)_{n}}{(a q, b q ; q)_{n}}(a b)^{2 n} q^{2 n^{2}} .
$$


Now

$$
\begin{aligned}
& \lim _{a, b \rightarrow 1} \frac{1}{(1-a)(1-b)}\left(\frac{\left(\frac{q}{a}, \frac{q}{b} ; q\right)_{\infty}}{\left(a b q, \frac{q}{a b}, q ; q\right)_{\infty}}-\frac{1}{(a b q)_{\infty}}\right) \\
& \quad=\lim _{a, b \rightarrow 1} \frac{1}{(1-a)(1-b)}\left(\frac{1}{(a b q)_{\infty}} \sum_{n=0}^{\infty} \frac{(a)_{n}(b)_{n}\left(\frac{q}{a b}\right)^{n}}{(q)_{n}^{2}}-\frac{1}{(a b q)_{\infty}}\right) \\
& \quad=\lim _{a, b \rightarrow 1} \frac{1}{(a b q)_{\infty}} \sum_{n=1}^{\infty} \frac{(a q)_{n-1}(b q)_{n-1}\left(\frac{q}{a b}\right)^{n}}{(q)_{n}^{2}}=\frac{1}{(q)_{\infty}} \sum_{n=1}^{\infty} \frac{q^{n}}{\left(1-q^{n}\right)^{2}}
\end{aligned}
$$

where we use Corollary 2.3 of [Andrews 1998] in the second equality.

Next we have

$$
\begin{aligned}
& \lim _{a, b \rightarrow 1} \frac{\left(\frac{q}{a}, \frac{q}{b} ; q\right)_{\infty}}{(1-a)(1-b)\left(a b q, \frac{q}{a b}, q ; q\right)_{\infty}} \sum_{n \neq 0} \frac{\left(1-a b q^{2 n}\right)}{(1-a b)} \frac{(a, b ; q)_{n}}{(b q, a q ; q)_{n}} q^{2 n^{2}}(a b)^{2 n} \\
& =\frac{1}{(q)_{\infty}} \lim _{a, b \rightarrow 1} \sum_{n \neq 0} \frac{\left(1-a b q^{2 n}\right) q^{2 n^{2}}(a b)^{2 n}}{(1-a b)\left(1-a q^{n}\right)\left(1-b q^{n}\right)} \\
& =\frac{1}{(q)_{\infty}} \lim _{b \rightarrow 1} \sum_{n=1}^{\infty}\left(\frac{\left(1-b q^{2 n}\right) b^{2 n} q^{2 n^{2}}}{(1-b)\left(1-q^{n}\right)\left(1-b q^{n}\right)}+\frac{\left(1-b q^{-2 n}\right) b^{-2 n} q^{2 n^{2}}}{(1-b)\left(1-q^{-n}\right)\left(1-b q^{-n}\right)}\right) \\
& =-\left.\frac{1}{(q)_{\infty}} \sum_{n=1}^{\infty} \frac{d}{d b}\right|_{b=1}\left(\frac{\left(1-b q^{2 n}\right) b^{2 n} q^{2 n^{2}}}{(1-b)\left(1-q^{n}\right)\left(1-b q^{n}\right)}+\frac{\left(q^{2 n}-b\right) b^{-2 n} q^{2 n^{2}}}{(1-b)\left(1-q^{n}\right)\left(b-q^{n}\right)}\right) \\
& =-\frac{4}{(q)_{\infty}} \sum_{n=1}^{\infty} \frac{n\left(1+q^{n}\right) q^{2 n^{2}}}{1-q^{n}}+\frac{2}{(q)_{\infty}} \sum_{n=1}^{\infty} \frac{q^{2 n^{2}+n}}{1-q^{n}}
\end{aligned}
$$

Comparing (6-3), (6-4), and (6-5) and multiplying by $(q)_{\infty} / 2$ we obtain the result.

\section{Lemma 6.3.}

$$
\begin{aligned}
\sum_{n=1}^{\infty} \frac{q^{n}}{\left(1-q^{n}\right)^{2}}-\sum_{n=1}^{\infty} \frac{q^{n}}{\left(1+q^{n}\right)^{2}} & =4 \sum_{n=1}^{\infty} \frac{q^{2 n}}{\left(1-q^{2 n}\right)^{2}} \\
& =\sum_{n=1}^{\infty} \frac{4 n\left(1+q^{2 n}\right) q^{2 n^{2}}}{1-q^{2 n}}+4 \sum_{n=1}^{\infty} \frac{q^{2 n^{2}+2 n}}{\left(1-q^{2 n}\right)^{2}}
\end{aligned}
$$


Proof. The first equality is immediate and follows by combining the initial two sums term by term. Finally,

$$
\begin{aligned}
\sum_{n=1}^{\infty} \frac{q^{2 n}}{\left(1-q^{2 n}\right)^{2}} & =\sum_{n, m=1}^{\infty} m q^{2 n m}=\sum_{m=1}^{\infty}\left(\sum_{n=1}^{m}+\sum_{m+1}^{\infty}\right) m q^{2 n m} \\
& =\sum_{n=1}^{\infty} \sum_{m=n}^{\infty} m q^{2 n m n}+\sum_{m=1}^{\infty} \frac{m q^{2 m^{2}+2 m}}{1-q^{2} m} \\
& =\sum_{n=1}^{\infty} \sum_{m=0}^{\infty}(m+n) q^{2 n(m+n)}+\sum_{m=1}^{\infty} \frac{m q^{2 m^{2}+2 m}}{1-q^{2 m}} \\
& =\sum_{n=1}^{\infty} \frac{q^{2 n^{2}+2 n}}{\left(1-q^{2 n}\right)^{2}}+\sum_{n=1}^{\infty} \frac{n q^{2 n^{2}}}{1-q^{2 n}}+\sum_{m=1}^{\infty} \frac{m q^{2 m^{2}+2 m}}{1-q^{2 m}} \\
& =\sum_{n=1}^{\infty} \frac{n q^{2 n^{2}}\left(1+q^{2 n}\right)}{1-q^{2 n}}+\sum_{n=1}^{\infty} \frac{q^{2 n^{2}+2 n}}{\left(1-q^{2 n}\right)^{2}} .
\end{aligned}
$$

We are now ready to prove the main result.

$q$-Series proof of Theorem 1.3. Comparing the statement of Theorem 1.3 with the assertion of Lemma 1, we see that the theorem is equivalent to the following:

$$
\begin{aligned}
& \sum_{n=1}^{\infty} \frac{q^{2 n^{2}+n}}{\left(1+q^{n}\right)^{2}}-2 \sum_{n=1}^{\infty} \frac{n q^{2 n^{2}}\left(1-q^{n}\right)}{1+q^{n}} \\
& \quad=\sum_{n=1}^{\infty} \frac{q^{n}}{\left(1+q^{n}\right)^{2}}+\sum_{n=1}^{\infty} \frac{(-1)^{n} n q^{n(n+1) / 2}}{1-q^{n}}
\end{aligned}
$$

Now in (6-6) we replace the final sum on the right-hand side by the negative of the right-hand side of the identity given by Lemma 6.2. Hence Theorem 1.3 is equivalent to the assertion that

$$
\begin{aligned}
& \sum_{n=1}^{\infty} \frac{q^{2 n^{2}+n}}{\left(1+q^{n}\right)^{2}}-2 \sum_{n=1}^{\infty} \frac{n q^{2 n^{2}}\left(1-q^{n}\right)}{1+q^{n}} \\
& \quad=\sum_{n=1}^{\infty} \frac{q^{n}}{\left(1+q^{n}\right)^{2}}-\sum_{n=1}^{\infty} \frac{q^{n}}{\left(1-q^{n}\right)^{2}}+2 \sum_{n=1}^{\infty} \frac{n q^{2 n^{2}}\left(1+q^{n}\right)}{1-q^{n}}+\sum_{n=1}^{\infty} \frac{q^{2 n^{2}+n}}{\left(1-q^{n}\right)^{2}}
\end{aligned}
$$

We now combine the first sum on the left-hand side with the final sum on the right-hand side and apply Lemma 6.3 to the first two sums on the right-hand side. 
Hence Theorem 1.3 is equivalent to the assertion that

$$
-4 \sum_{n=1}^{\infty} \frac{q^{2 n^{2}+2 n}}{\left(1-q^{2 n}\right)^{2}}-4 \sum_{n=1}^{\infty} \frac{n q^{2 n^{2}}\left(1-q^{n}\right)}{1+q^{n}}=\sum_{n=1}^{\infty} \frac{q^{n}}{\left(1+q^{n}\right)^{2}}-\sum_{n=1}^{\infty} \frac{q^{n}}{\left(1-q^{n}\right)^{2}},
$$

which is merely a restatement of Lemma 6.3.

\section{Modular proof of Theorem 1.3}

In this section we establish Theorem 1.3 by computing the modular transformations of the right hand side and demonstrate that they match those of the left hand side. Finally checking that enough of the Fourier coefficients are equal yields the result. "Modular" proof of Theorem 1.3. We use Theorem 2.6 together with (2-12) to find

$$
\begin{aligned}
\widehat{\mu}(u, u ; \tau)=\mu(u, u ; \tau)+\frac{i}{2} R(0 ; \tau) & =\mu(u, u ; \tau)-\frac{i}{2} \int_{-\bar{\tau}}^{i \infty} \frac{g_{\frac{1}{2}, \frac{1}{2}}(z)}{\sqrt{-i(z+\tau)}} d z \\
& =\mu(u, u ; \tau)+\frac{1}{2} \int_{-\bar{\tau}}^{i \infty} \frac{\eta(z)^{3}}{\sqrt{-i(z+\tau)}} d z .
\end{aligned}
$$

Combining this with the definition of $\widehat{f}_{1}$ in Theorem 3.2 and the definition of $\widehat{E}_{2}$ in (2-6), we see that if we define

$$
M(u ; \tau):=f_{1}(\tau)+i \eta(\tau)^{3} \mu(u, u ; \tau)-\frac{1}{12} E_{2}(\tau),
$$

then this also equals $\widehat{f}_{1}(\tau)+i \eta(\tau)^{3} \widehat{\mu}(u, u ; \tau)-\frac{1}{12} \widehat{E}_{2}(\tau)$. Now using the transformation properties of $\widehat{f}_{1}$ from Theorem 3.2, $\widehat{\mu}$ from Theorem 2.5, and $\widehat{E}_{2}$, we get that $M$ transforms as

$$
M\left(\frac{u}{c \tau+d} ; \frac{a \tau+b}{c \tau+d}\right)=(c \tau+d)^{2} M(u ; \tau)
$$

for all $\left(\begin{array}{ll}a & b \\ c & d\end{array}\right) \in \mathrm{SL}_{2}(\mathbb{Z})$. Further, by the first part of Theorem 2.5 we have the elliptic transformation property $M(u+k \tau+l ; \tau)=M(u ; \tau)$, for all $k, l \in \mathbb{Z}$. Using these we easily see that

$$
m(\tau):=\left(\begin{array}{c}
M\left(\frac{1}{2} ; \tau\right) \\
M(\tau / 2 ; \tau) \\
M((\tau+1) / 2 ; \tau)
\end{array}\right)
$$

transforms as a vector-valued modular form of weight 2 on $\mathrm{SL}_{2}(\mathbb{Z})$ :

$$
m(\tau+1)=\left(\begin{array}{lll}
1 & 0 & 0 \\
0 & 0 & 1 \\
0 & 1 & 0
\end{array}\right) m(\tau) \text { and } m\left(-\frac{1}{\tau}\right)=\tau^{2}\left(\begin{array}{lll}
0 & 1 & 0 \\
1 & 0 & 0 \\
0 & 0 & 1
\end{array}\right) m(\tau) .
$$

From this we see that $M\left(\frac{1}{2} ; \tau\right)$ transforms as a modular form of weight 2 on $\Gamma_{0}(2)$. 
On the other hand,

$$
g(\tau):=\left(\begin{array}{c}
E_{2}(\tau)-2 E_{2}(2 \tau) \\
E_{2}(\tau)-\frac{1}{2} E_{2}(\tau / 2) \\
E_{2}(\tau)-\frac{1}{2} E_{2}((\tau+1) / 2)
\end{array}\right)=\left(\begin{array}{c}
\widehat{E}_{2}(\tau)-2 \widehat{E}_{2}(2 \tau) \\
\widehat{E}_{2}(\tau)-\frac{1}{2} \widehat{E}_{2}(\tau / 2) \\
\widehat{E}_{2}(\tau)-\frac{1}{2} \widehat{E}_{2}((\tau+1) / 2)
\end{array}\right)
$$

satisfies the same modular transformation properties as $m$. Further we get directly from the definitions

$$
g(\tau)=\left(\begin{array}{c}
-1+O(q) \\
\frac{1}{2}+O\left(q^{\frac{1}{2}}\right) \\
\frac{1}{2}+O\left(q^{\frac{1}{2}}\right)
\end{array}\right) \quad \text { and } \quad m(\tau)=\left(\begin{array}{c}
\frac{1}{6}+O(q) \\
-\frac{1}{12}+O\left(q^{\frac{1}{2}}\right) \\
-\frac{1}{12}+O\left(q^{\frac{1}{2}}\right)
\end{array}\right)
$$

and so

$$
m(\tau)+\frac{1}{6} g(\tau)=\left(\begin{array}{c}
O(q) \\
O\left(q^{\frac{1}{2}}\right) \\
O\left(q^{\frac{1}{2}}\right)
\end{array}\right)
$$

If we take the product of the three components of $m+\frac{1}{6} g$, then we get a holomorphic cusp form on $\mathrm{SL}_{2}(\mathbb{Z})$ of weight 6 , and hence this equals zero. This then implies that all three components are zero and so we get $m+\frac{1}{6} g=0$.

The first component equals

$$
\begin{aligned}
\frac{1}{6}\left(-1-24 \sum_{n>0} \sigma_{1}^{\text {odd }}(n) q^{n}\right) & -\frac{1}{12} E_{2}(\tau) \\
& +\sum_{n \neq 0} \frac{(-1)^{n+1} n q^{n(n+1) / 2}}{1-q^{n}}+2 \frac{(q)_{\infty}^{2}}{(-q)_{\infty}^{2}} \sum_{n \in \mathbb{Z}} \frac{q^{n(n+1) / 2}}{1+q^{n}}
\end{aligned}
$$

where we have used

$$
\vartheta\left(\frac{1}{2} ; \tau\right)=-2 q^{\frac{1}{8}}(q)_{\infty}(-q)_{\infty}^{2} \quad \text { and } \quad \sigma_{1}^{\text {odd }}(n)=\sum_{\substack{d \mid n \\ d \text { odd }}} d .
$$

Using (4-1), (4-2), and (3-4) to rewrite the Eisenstein series terms gives the result.

Remark. The calculations above show that $M(u ; \tau)$ is a multiple of the Weierstrass $\wp$-function. Thus the conclusion follows from known facts about $\wp$ at half periods. We have included the details for completeness. 


\section{Holomorphic projection}

In this section we demonstrate that the function

$$
B(\tau)=\frac{1}{\eta(\tau)^{3}} \sum_{n \neq 0} \frac{(-1)^{n} n q^{n(n+1) / 2}}{1-q^{n}}
$$

arises from the holomorphic projection of $\eta^{3}(\tau)\left(\eta^{3}(\tau)\right)^{*}$. The holomorphic projection operator is the unique linear map $\pi_{\mathrm{hol}}=\pi_{\mathrm{hol}, k}$ mapping the space of nonholomorphic modular forms of weight $k$ to the space of cusp forms of weight $k$ and level $N$ satisfying $(h, \Phi)=(h, \pi(\Phi))$ for all cusp forms $h$ of weight $k$ and level $N$ and $(\cdot, \cdot)$ is the Petersson inner product.

If $\Phi(\tau):=\sum_{n \in \mathbb{Z}} a_{n}(y) q^{n}$ is a modular form of weight $k$, not necessarily holomorphic, on $\mathrm{SL}_{2}(\mathbb{Z})$ such that for $\tau \rightarrow i \infty$ there exists an $\epsilon>0$ with

$$
\left(\left.\Phi\right|_{k} \gamma\right)(\tau)=O\left(y^{-\epsilon}\right)
$$

then $\pi_{\text {hol }}(\Phi)(\tau):=\sum_{n=1}^{\infty} c_{n}(\Phi) q^{n}$ with

$$
c_{n}(\Phi):=\frac{(4 \pi n)^{k-1}}{\Gamma(k-1)} \int_{0}^{\infty} a_{n}(y) e^{-4 \pi n y} y^{k-2} d y .
$$

See for instance [Sturm 1980] or Proposition 5.1 of [Gross and Zagier 1986].

Proposition 6.2 of [Gross and Zagier 1986] (see also Proposition 11 of [Coates 1986]) suggests that $\left(1 / \eta^{3}\right) \pi_{\text {hol }}\left(\eta^{3}\left(\eta^{3}\right)^{*}\right)$ is, after the addition of an Eisenstein series, the holomorphic part of a harmonic weak Maass form (see [Ono 2008] for discussion of holomorphic parts of harmonic weak Maass forms). Precisely, we have the following result.

Proposition 8.1. With the notation from above,

$$
\pi_{\mathrm{hol}}\left(\eta^{3}\left(\eta^{3}\right)^{*}\right)(\tau)=\sum_{n \neq 0} \frac{(-1)^{n} n q^{n(n+1) / 2}}{1-q^{n}} .
$$

Proof. For simplicity let $g(\tau)=\eta^{3}(\tau)$. From (2-3) we have

$$
g(\tau)=\sum_{n=1}^{\infty}\left(\frac{-4}{n}\right) n q^{n^{2} / 8} .
$$

Thus

$$
(g(\tau))^{*}=\pi^{-\frac{1}{2}} \sum_{n=1}^{\infty}\left(\frac{-4}{n}\right) \Gamma\left(\frac{1}{2} ; \frac{\pi n^{2} y}{2}\right) q^{-n^{2} / 8},
$$


so that

$$
g(\tau) g^{*}(\tau)=\pi^{-\frac{1}{2}} \sum_{n, m>0} m\left(\frac{-4}{n m}\right) \Gamma\left(\frac{1}{2} ; \frac{\pi n^{2} y}{2}\right) q^{\left(m^{2}-n^{2}\right) / 8}
$$

With $\ell=m^{2}-n^{2}$ we need to compute

$$
\begin{aligned}
\int_{0}^{\infty} & \Gamma\left(\frac{1}{2} ; \frac{\pi n^{2} y}{2}\right) e^{-\frac{\pi \ell y}{2}} d y \\
& =\int_{0}^{\infty} \int_{\frac{\pi n^{2} y}{2}}^{\infty} e^{-t} t^{\frac{1}{2}} e^{-\frac{\pi \ell y}{2}} \frac{d t}{t} d y=\int_{0}^{\infty} \int_{1}^{\infty} e^{-\left(w n^{2}+\ell\right) \frac{\pi}{2} y}(y w)^{\frac{1}{2}} \frac{d w}{w} d y \\
& =n \sqrt{\frac{\pi}{2}} \int_{1}^{\infty} w^{\frac{1}{2}} \frac{d w}{w} \int_{0}^{\infty} e^{-\left(w n^{2}+\ell\right) \frac{\pi}{2} y} y^{\frac{3}{2}} \frac{d y}{y} \\
& =\frac{n}{\sqrt{\pi}} \int_{1}^{\infty} \frac{1}{w^{\frac{1}{2}}\left(w n^{2}+\ell\right)^{\frac{3}{2}}} d w=\frac{n}{\sqrt{\pi}}\left(\left.\frac{2 \sqrt{w}}{\ell \sqrt{w n^{2}+\ell}}\right|_{1} ^{\infty}\right) \\
& =\frac{2 n}{\sqrt{\pi} \ell}\left(\frac{1}{n}-\frac{1}{\sqrt{n^{2}+\ell}}\right)=\frac{2}{\sqrt{\pi} m(m+n)},
\end{aligned}
$$

where we use $\int_{0}^{\infty} e^{-\alpha y} y^{1 / 2} d y / y=\alpha^{-3 / 2} \sqrt{\pi} / 2$ in the third equality.

Inserting this into (8-2) gives

$$
\pi_{\mathrm{hol}}\left(g g^{*}\right)(\tau)=\sum_{m>n>0}\left(\frac{-4}{n m}\right)(m-n) q^{\left(m^{2}-n^{2}\right) / 8} .
$$

Now $(-4 / n)(-4 / m)=0$ unless both $n$ and $m$ are odd, in which case we have $n=2 a+1$ and $m=2 b+1$. Thus $(-4 / n m)=(-1)^{a+b}$ and we have

$$
\begin{aligned}
\pi_{\mathrm{hol}}\left(g g^{*}\right)(\tau) & =2 \sum_{a>b \geq 0}(-1)^{a+b}(a-b) q^{\frac{a^{2}+a-b^{2}-b}{2}} \\
& =2 \sum_{b \geq 0, h>0}(-1)^{h} h q^{\frac{h^{2}+h}{2}+b h}=2 \sum_{h>0} \frac{(-1)^{h} h q^{h(h+1) / 2}}{1-q^{h}},
\end{aligned}
$$

which yields the result.

\section{Proof of Theorem 1.4}

In this section we compute an asymptotic expansion for $V(n)$. We follow a circle method argument used by Bringmann and Mahlburg [2011] (see also [Bringmann and Mahlburg 2013]) to calculate an asymptotic for coefficients of mixed modular forms. It is convenient to work with the two variable rank generating function for concave compositions given in (1-7) and (1-8). We begin with the proof of Theorem 1.6. 
Proof of Theorem 1.6. The identity for $v_{d}(x, q)$ follows from an application of (3.6) of [Andrews 1981] and then (13.3) and (6.1) of [Fine 1988]. The second identity is a corollary of Theorem 4 of [Choi 2011].

9A. Circle method and the proof of Theorem 1.4. By Cauchy's theorem we have

$$
V(n-1)=\frac{1}{2 \pi i} \int_{C} \frac{q v(q)}{q^{n+1}} d q,
$$

where $C$ is an arbitrary path inside the unit circle that loops around 0 in the counterclockwise direction. We choose the circle with radius $r=e^{-\pi / N^{2}}$ with $N:=\left\lfloor n^{1 / 2}\right\rfloor$, and use the parametrization $q=e^{-2 \pi / N^{2}+2 \pi i t}$ with $0 \leq t \leq 1$. As usual in the circle method, we define

$$
\vartheta_{h, k}^{\prime}:=\frac{1}{k\left(k_{1}+k\right)} \quad \text { and } \quad \vartheta_{h, k}^{\prime \prime}:=\frac{1}{k\left(k_{2}+k\right)},
$$

where $h_{1} / k_{1}<h / k<h_{2} / k_{2}$ are adjacent Farey fractions in the Farey sequence of order $N:=\left\lfloor n^{1 / 2}\right\rfloor$. So $1 /\left(k+k_{j}\right) \leq 1 /(N+1)$ for $j=1,2$. Next, decompose the path of integration into paths along the Farey arcs $-\vartheta_{h, k}^{\prime} \leq \Phi \leq \vartheta_{h, k}^{\prime \prime}$, where $\Phi$ is defined by $z=\left(k / N^{2}\right)-k i \Phi$ with $-\vartheta_{h, k}^{\prime} \leq \Phi \leq \vartheta_{h, k}^{\prime \prime}$ and $0 \leq h \leq k \leq N$ with $(h, k)=1$. Hence,

$$
V(n-1)=\sum_{\substack{1 \leq k<N \\(h, k)=1}} e^{-\frac{2 \pi i h n}{k}} \int_{-\vartheta_{h, k}^{\prime}}^{\vartheta_{h, k}^{\prime \prime}} v\left(e^{\frac{2 \pi i}{k}(h+i z)}\right) e^{\frac{2 \pi i}{k}(h+i z)} e^{\frac{2 \pi n z}{k}} d \Phi .
$$

For computing the asymptotic nature of $v\left(e^{(2 \pi i / k)(h+i z)}\right)$ as $z \rightarrow 0$, the useful form of Theorem 1.6 is

$$
q v(x, q)=q^{\frac{1}{12}} \frac{\eta(\tau)}{2 \sin (\pi u) \vartheta(u ; \tau)} R(u, \tau)-i q^{\frac{1}{8}} \mu(u,-u ; \tau),
$$

where we have set $q=e^{2 \pi i \tau}, x=e^{2 \pi i u}$, and

$$
R(u, \tau)=\sum_{n=0}^{\infty} \frac{q^{n^{2}}}{(x q)_{n}\left(x^{-1} q\right)_{n}}
$$

is the Rank generating function; see [Ono 2008]. The idea is to compute the asymptotics as $z \rightarrow 0^{+}$of the two variable version of the generating function and then set $u=0$. We are interested in exponential growth and will freely ignore terms once they are determined to have smaller growth.

The proof of Proposition 3.5 of [Bringmann et al. 2012] gives the following asymptotic evaluation for $R(u, \tau)$. Let $\widetilde{h} \in\{-1,0,1\}$ defined by $\widetilde{h} \equiv h(\bmod 3)$. 
Moreover, if $0 \leq \ell<k$, then we write

$$
\begin{gathered}
\quad \alpha^{ \pm}(\ell, k):=\frac{1}{k}\left( \pm \frac{1}{3}-\left(\ell-\frac{k-1}{2}\right)\right), \quad \tilde{k}= \begin{cases}3 k & \text { if } 3 \nmid k, \\
k / 3 & \text { if } 3 \mid k,\end{cases} \\
\tilde{H}^{ \pm}(u, h, \ell, k ; z) \\
:= \pm e^{\frac{\tilde{h}^{2} \pi}{3 k z}} \xi_{\ell}\left(\frac{3 h}{(3, k)}, \frac{k}{(3, k)}\right) H\left(\frac{(3, k) i u}{z}+\alpha^{ \pm}\left(\ell, \frac{k}{(3, k)}\right) \mp \frac{\tilde{h} i}{k z} ; \frac{i}{\widetilde{k} z}\right),
\end{gathered}
$$

where

$$
\xi_{\ell}(h, k):=(-1)^{\ell+1} e^{-\frac{\pi i h}{4 k}(2 \ell+1)^{2} \mp \frac{\pi i}{k}\left(\frac{\tilde{h}-h}{3}\right)(2 \ell+1)+\frac{2 \pi i \tilde{h}}{9 k}} .
$$

Proposition 9.1. For $\tau=(1 / k)(h+i z)$ with $0 \leq h<k$ with $(h, k)=1$ set $q=e^{(2 \pi i / k)(h+i z)}$ and $q_{1}=e^{(2 \pi i / k)\left([-h]_{k}+(i / z)\right)}$. With this notation, we have

$$
\begin{aligned}
& R(u, \tau)=-i^{\frac{3}{2}} \chi^{-1}\left(h,[-h]_{k}, k\right)\left(q q_{1}^{-1}\right)^{\frac{1}{24}} z^{-\frac{1}{2}} e^{\frac{3 k \pi u^{2}}{z}} \frac{\sin (\pi u)}{\sinh \left(\frac{\pi u}{z}\right)} \\
& +\sum_{\ell=1}^{\infty} a_{\ell}(z) \frac{(2 \pi i u)^{\ell}}{\ell !}-\frac{i \sin (\pi u)}{\sqrt{\widetilde{k} z}} e^{-\frac{\pi z}{12 k}-\frac{\pi i h}{4 k}+\frac{3 \pi k u^{2}}{z}} \sum_{ \pm} \sum_{\ell=0}^{\frac{k}{(3, k)}-1} \tilde{H}^{ \pm}(u, h, \ell, k ; z)
\end{aligned}
$$

with $a_{\ell}(z) \ll_{\ell}|z|^{1-\ell} e^{-(23 \pi /(12 k)) \operatorname{Re}(1 / z)}$.

Additionally, we have

$\frac{q^{\frac{1}{12}} \eta(\tau)}{\vartheta(u ; \tau)}=q^{\frac{1}{12}} \chi^{-2}\left(h,[-h]_{k}, k\right) e^{k \pi u^{2} / z} \frac{\eta\left(\tau^{\prime}\right)}{\vartheta\left(\frac{i u}{z} ; \tau^{\prime}\right)}=\frac{q^{\frac{1}{12}} q_{1}^{\frac{1}{24}}\left(1+O\left(q_{1}\right)\right) e^{k \pi u^{2} / z}}{\chi^{2}\left(h,[-h]_{k}, k\right) \vartheta\left(\frac{i u}{z} ; \tau^{\prime}\right)}$.

Thus

$$
\begin{aligned}
& \frac{q^{\frac{1}{12}} \eta(\tau)}{2 \sin (\pi u) \vartheta(u ; \tau)} R(u, \tau) \\
& =-i^{\frac{3}{2}} \chi^{-3}\left(h,[-h]_{k}, k\right) q^{\frac{1}{8}} \frac{z^{-\frac{1}{2}} e^{\frac{4 \pi k u^{2}}{z}}}{2 \sinh \left(\frac{\pi u}{z}\right) \vartheta\left(\frac{i u}{z} ; \tau^{\prime}\right)}+O\left(e^{-\frac{\alpha}{k} \operatorname{Re}\left(\frac{1}{z}\right)}\right) \\
& -i \chi^{-2}\left(h,[-h]_{k}, k\right) \frac{q^{\frac{1}{12}} e^{-\frac{\pi z}{12 k}-\frac{\pi i h}{4 k}} q_{1}^{\frac{1}{24}}\left(1+O\left(q_{1}\right)\right)}{2 \sqrt{\tilde{k} z} \vartheta\left(\frac{i u}{z} ; \tau^{\prime}\right)} \\
& \times \sum_{ \pm}^{\frac{k}{(3, k)}-1} \sum_{\ell=0}^{\frac{k}{T^{\prime}}} \tilde{H}^{ \pm}(u, h, \ell, k ; z)
\end{aligned}
$$

for some $\alpha>0$ independent of $k$ as $z \rightarrow 0$ and $u \rightarrow 0$.

Turning to the other term we have the following proposition. 
Proposition 9.2. Let $\tau=\frac{1}{k}(h+i z)$ and $\tau^{\prime}=(1 / k)\left([-h]_{k}+(i / z)\right)$ then

$$
\begin{aligned}
\mu(u,-u ; \tau) & =i^{\frac{1}{2}} \chi^{-3}\left(h,[-h]_{k}, k\right) z^{-\frac{1}{2}} e^{\frac{4 \pi k u^{2}}{z}} \mu\left(\frac{i u}{z},-\frac{i u}{z} ; \tau^{\prime}\right) \\
& +\frac{(-1)^{h+1} i}{2} e^{\frac{4 \pi k u^{2}}{z}} \sum_{\ell=0}^{k-1} e^{-\frac{\pi i}{k}\left(\ell+\frac{1}{2}\right)^{2}} H\left(\frac{2 i u}{z}-\frac{1}{k}\left(\ell-\frac{k-1}{2}\right) ; \frac{i}{k z}\right) .
\end{aligned}
$$

Proof. Theorem 2.5 implies that

$$
\begin{aligned}
\mu(u,-u ; \tau)= & \chi^{-3}\left(h,[-h]_{k}, k\right) \sqrt{\frac{i}{z}} e^{\frac{4 \pi k u^{2}}{z}} \mu\left(\frac{i u}{z},-\frac{i u}{z} ; \tau^{\prime}\right) \\
& +\frac{i}{2} \chi^{-3}\left(h,[-h]_{k}, k\right) \sqrt{\frac{i}{z}} e^{\frac{4 \pi k u^{2}}{z}} R\left(\frac{2 i u}{z} ; \tau^{\prime}\right)-\frac{i}{2} R(2 u ; \tau) .
\end{aligned}
$$

Propositions 2.3 and 2.4 yield

$$
\begin{aligned}
& R(2 u ; \tau)=-\sum_{\ell=0}^{k-1} e^{-\frac{\pi i}{k}(h+i z)\left(\ell-\frac{k-1}{2}\right)^{2}-4 \pi i u\left(\ell-\frac{k-1}{2}\right)} e^{-\pi i\left(\ell-\frac{k-1}{2}\right)-\frac{\pi i k h}{4}} \\
& \times(-1)^{\left(\ell-\frac{k-1}{2}\right)(h+1)} \frac{e^{\frac{\pi}{k z}\left(2 u k+\left(\ell-\frac{k-1}{2}\right) i z\right)^{2}}}{\sqrt{k z}} \\
& \times\left(R\left(\frac{2 i u}{z}-\frac{1}{k}\left(\ell-\frac{k-1}{2}\right) ; \frac{i}{k z}\right)-H\left(\frac{2 i u}{z}-\frac{1}{k}\left(\ell-\frac{k-1}{2}\right) ; \frac{i}{k z}\right)\right) .
\end{aligned}
$$

The nonholomorphic $R$-functions above will exactly cancel with the other term of (9-6); this can be shown as in [Bringmann and Mahlburg 2013; Bringmann et al. 2012]. Simplifying the factors and multiplying the $H$-functions gives the result.

We will evaluate the asymptotic nature of the two terms on the right hand side of Proposition 9.2. First consider the terms with the $H$-function. We have

$$
H\left(\frac{2 i u}{z}-\frac{1}{k}\left(\ell-\frac{k-1}{2}\right) ; \frac{i}{k z}\right)=O\left(\int_{-\infty}^{\infty} e^{-\frac{\pi x^{2}}{k} \operatorname{Re}\left(\frac{1}{z}\right)} d x\right)=O\left(\sqrt{k} \operatorname{Re}\left(\frac{1}{z}\right)^{-\frac{1}{2}}\right)
$$

as $u \rightarrow 0$ and $z \rightarrow 0$. Next turning to the other term, we have

$$
\mu\left(\frac{i u}{z},-\frac{i u}{z} ; \tau^{\prime}\right)=\frac{1}{2 \sinh \left(\frac{\pi u}{z}\right) \vartheta\left(-\frac{i u}{z} ; \tau^{\prime}\right)}+\frac{e^{-\frac{\pi u}{z}}}{\vartheta\left(-\frac{i u}{z} ; \tau^{\prime}\right)} \sum_{n \neq 0} \frac{(-1)^{n} q_{1}^{\frac{n(n+1)}{2}} e^{\frac{2 \pi n u}{z}}}{1-e^{-\frac{2 \pi u}{z}} q_{1}^{n}} .
$$

The summation is $O\left(u q_{1}\right)$, since it is 0 when $u=0$ by symmetry. Consequently, using Lemma 5.2 we have $\vartheta\left(-i u / z ; \tau^{\prime}\right)^{-1}=O\left((z / u) q_{1}^{-1 / 8}\right)$, and we see that the term with the summation is $O\left(q_{1}^{7 / 8}\right)=O\left(e^{-7 \pi /(4 k z)}\right)$ after setting $u=0$. So it is 
of exponential decay and will not contribute to the asymptotic expansion of our generating function. Hence

$$
\begin{aligned}
-i q^{\frac{1}{8}} \mu(u,-u ; \tau) & \\
= & -i^{\frac{3}{2}} \chi^{-3}\left(h,[-h]_{k}, k\right) q^{\frac{1}{8}} z^{-\frac{1}{2}} e^{4 \pi k u^{2} / z} \mu\left(\frac{i u}{z},-\frac{i u}{z} ; \tau^{\prime}\right)+O_{k}(1) \\
& =\frac{i^{\frac{3}{2}} q^{\frac{1}{8}} \chi^{-3}\left(h,[-h]_{k}, k\right) z^{-\frac{1}{2}} e^{4 \pi k u^{2} / z}}{2 \sinh \left(\frac{\pi u}{z}\right) \vartheta\left(\frac{i u}{z} ; \tau^{\prime}\right)}+O\left(z^{-\frac{1}{2}} q_{1}^{-\frac{7}{8}}\right)+O_{k}(1)
\end{aligned}
$$

as $u \rightarrow 0$ when $z$ is chosen as in the circle method calculations.

Using (9-2), (9-5), and (9-7) we see that as $u \rightarrow 0$ and $z \rightarrow 0$ we have the following asymptotic evaluation for $v(q)$ with $q=e^{(2 \pi i / k)(h+i z)}$.

$$
\begin{aligned}
& q v(q) \sim-i \chi^{-2}\left(h,[-h]_{k}, k\right) \\
& \qquad \times \frac{q^{\frac{1}{12}} e^{-\frac{\pi z}{12 k}-\frac{\pi i h}{4 k}} q_{1}^{\frac{1}{24}}\left(1+O\left(q_{1}\right)\right)}{2 \sqrt{\tilde{k} z} \vartheta\left(\frac{i u}{z} ; \tau^{\prime}\right)} \sum_{ \pm} \sum_{\ell=0}^{\frac{k}{(3, k)}-1} \tilde{H}^{ \pm}(u, h, \ell, k ; z) .
\end{aligned}
$$

It is evident that the largest growth comes from the case when $k=1$ and thus the largest contribution to (9-1) will come from the case $k=1$. In this case we set

$$
\begin{aligned}
\sum_{ \pm} \tilde{H}^{ \pm}(u, 0,0,1 ; z)= & -H\left(\frac{i u}{z}+\frac{1}{3} ; \frac{i}{3 z}\right)+H\left(\frac{i u}{z}-\frac{1}{3} ; \frac{i}{3 z}\right) \\
& =-\int_{-\infty}^{\infty} \frac{e^{-\pi x^{2} /(3 z)}}{\cosh (\pi x)} e^{-2 \pi i x u / z}\left(e^{-2 \pi x / 3}-e^{2 \pi x / 3}\right) d x \\
& =-2 i \int_{-\infty}^{\infty} e^{-\pi x^{2} /(3 z)} \sin \left(\frac{2 \pi x u}{z}\right) \frac{\sinh \left(\frac{2 \pi x}{3}\right)}{\cosh (\pi x)} d x \\
& =-2 i \cdot \frac{2 \pi u}{z} \int_{-\infty}^{\infty} x e^{-\pi x^{2} /(3 z)} \frac{\sinh \left(\frac{2 \pi x}{3}\right)}{\cosh (\pi x)} d x+O\left(u^{3}\right)
\end{aligned}
$$

Therefore, with $q=e^{-2 \pi z}$ we have

$$
\begin{aligned}
q v(q) \sim \lim _{u \rightarrow 0}-e^{-\frac{\pi z}{4}} e^{-\frac{\pi}{12 z}} \frac{1}{2 \sqrt{3 z \vartheta\left(\frac{i u}{z} ; \tau^{\prime}\right)}} \\
\quad \times\left(-\frac{4 \pi i u}{z} \int_{-\infty}^{\infty} x e^{-\frac{\pi x^{2}}{3 z}} \frac{\sinh \left(\frac{2 \pi x}{3}\right)}{\cosh (\pi x)} d x\right) \\
\sim \frac{e^{\frac{\pi}{6}\left(\frac{1}{z}-\frac{3}{2} z\right)}}{\sqrt{3 z}} \int_{-\infty}^{\infty} x e^{-\frac{\pi x^{2}}{3 z}} \frac{\sinh \left(\frac{2 \pi x}{3}\right)}{\cos (\pi x)} d x .
\end{aligned}
$$


Therefore, we have

$$
\begin{aligned}
V(n-1) & \sim-\int_{-\vartheta_{0,1}^{\prime}}^{\vartheta_{0,1}^{\prime \prime}} \frac{e^{\frac{\pi}{6}\left(\frac{1}{z}+\left(12 n-\frac{3}{2}\right) z\right)}}{\sqrt{3 z}} \int_{-\infty}^{\infty} x e^{-\frac{\pi x^{2}}{3 z}} \frac{\sinh \left(\frac{2 \pi x}{3}\right)}{\cosh (\pi x)} d x d \Phi \\
& \sim-\frac{1}{\sqrt{3}} \int_{-\infty}^{\infty} x \frac{\sinh \left(\frac{2 \pi x}{3}\right)}{\cosh (\pi x)} \int_{-\frac{1}{N}}^{\frac{1}{N}} z^{-\frac{1}{2}} e^{\frac{\pi}{6}\left(\left(1-2 x^{2}\right) \frac{1}{z}+\left(12 n-\frac{3}{2}\right) z\right)} d \Phi d x
\end{aligned}
$$

where $z=\left(1 / N^{2}\right)-i \Phi$ and we symmetrize the integral by using

$$
\int_{-\vartheta_{0,1}^{\prime}}^{\vartheta_{0,1}^{\prime \prime}}=\int_{-\frac{1}{N}}^{\frac{1}{N}}-\int_{-\frac{1}{N}}^{-\vartheta_{0,1}^{\prime}}-\int_{\vartheta_{0,1}^{\prime \prime}}^{\frac{1}{N}}
$$

and $\sim$ represents the asymptotic expansion with respect to $n$. The final two sums contribute a polynomially bounded error with respect to $N$. We handle the resulting integral with respect to $\Phi$ exactly as in Proposition 3.2 of [Bringmann and Mahlburg 2011] (see Lemma 4.2 of [Rhoades 2012] for an analogous calculation). This results in

$$
\begin{aligned}
\int_{-\frac{1}{N}}^{\frac{1}{N}} z^{-\frac{1}{2}} e^{\frac{\pi}{6}\left(\left(1-2 x^{2}\right) \frac{1}{z}+\left(12 n-\frac{3}{2}\right) z\right)} d \Phi & \\
= & \left.-2 \pi\left(\frac{1-2 x^{2}}{12 n-\frac{3}{2}}\right)^{\frac{1}{4}} I_{\frac{1}{2}}\left(\frac{\pi}{3} \sqrt{\left(12 n-\frac{3}{2}\right)\left(1-2 x^{2}\right.}\right)\right) \\
& \left.=-2 \sqrt{6}\left(12 n-\frac{3}{2}\right)^{-\frac{1}{2}} \sinh \left(\frac{\pi}{3} \sqrt{\left(12 n-\frac{3}{2}\right)\left(1-2 x^{2}\right.}\right)\right)
\end{aligned}
$$

Therefore,

$$
\begin{aligned}
V(n-1) & \sim \frac{2 \sqrt{2}}{\left(12 n-\frac{3}{2}\right)^{\frac{1}{2}}} \int_{-\infty}^{\infty} x \frac{\sinh \left(\frac{2 \pi x}{3}\right)}{\cosh (\pi x)} \sinh \left(\frac{\pi}{3} \sqrt{\left(12 n-\frac{3}{2}\right)\left(1-2 x^{2}\right)}\right) d x \\
& \sim \frac{2 \sqrt{2}}{\left(12 n-\frac{3}{2}\right)^{\frac{1}{2}}} \int_{-\frac{1}{\sqrt{2}}}^{\frac{1}{\sqrt{2}}} x \frac{\sinh \left(\frac{2 \pi x}{3}\right)}{\cosh (\pi x)} \sinh \left(\frac{\pi}{3} \sqrt{\left(12 n-\frac{3}{2}\right)\left(1-2 x^{2}\right)}\right) d x \\
& \sim \frac{2 \sqrt{2}}{\left(12 n-\frac{3}{2}\right)^{\frac{1}{2}}} \int_{0}^{\frac{1}{\sqrt{2}}} x \frac{\sinh \left(\frac{2 \pi x}{3}\right)}{\cosh (\pi x)} \exp \left(\frac{\pi}{3} \sqrt{\left(12 n-\frac{3}{2}\right)\left(1-2 x^{2}\right)}\right) d x
\end{aligned}
$$

where the second equality follows because

$$
\sinh \left(\frac{\pi}{3} \sqrt{\left(12 n-\frac{3}{2}\right)\left(1-2 x^{2}\right)}\right)=O(1) \quad \text { for }|x|>\frac{1}{\sqrt{2}} .
$$


We have the following lemma which gives the asymptotic expansion of the remaining integral.

Lemma 9.3. Let $\alpha_{k}$ for $k \geq 1$ be defined by

$$
\sum_{k=1}^{\infty} \alpha_{k} x^{2 k}=\exp \left(\sqrt{1-2 x^{2}}-1+x^{2}\right) \times \frac{\sinh \left(\frac{2 \pi x}{3}\right)}{\cosh (\pi x)} .
$$

As $y \rightarrow \infty$ we have

$$
\int_{0}^{\frac{1}{\sqrt{2}}} f e^{y \sqrt{1-2 x^{2}}} x \frac{\sinh \left(\frac{2 \pi x}{3}\right)}{\cosh (\pi x)} \sim e^{y} \frac{\sqrt{\pi}}{2 \sqrt{y}} \sum_{t=1}^{\infty} \frac{(2 t-1) ! !}{2^{2 t}} \alpha_{t} \frac{1}{y^{t}} .
$$

From (9-8) and Lemma 9.3 we have

$$
V(n-1) \sim \frac{\sqrt{6}}{\left(12 n-\frac{3}{2}\right)^{\frac{3}{4}}} \exp \left(\frac{\pi}{3} \sqrt{12 n-\frac{3}{2}}\right) \sum_{t=1}^{\infty} \frac{(2 t-1) ! ! 3^{t}}{2^{2 t} \pi^{t}} \alpha_{t}\left(12 n-\frac{3}{2}\right)^{-t / 2},
$$

which gives Theorem 1.4.

Note we have

$$
\begin{aligned}
\sum_{k=1}^{\infty} \alpha_{k} x^{2 k}=\frac{2 \pi}{3} x^{2}-\frac{23}{81} \pi^{3} x^{4}- & \frac{4860 \pi-1681 \pi^{5}}{14580} x^{6} \\
& +\frac{1837080 \pi+257543 \pi^{7}-782460 \pi^{3}}{5511240} x^{8}+\cdots
\end{aligned}
$$

\section{Proof of Theorem 1.5}

In this section we compute an asymptotic for $V_{d}(n)$. We follow the standard circle method set-up as in Section 9. As above, we have

$$
V_{d}(n)=\sum_{\substack{1 \leq k<N \\(h, k)=1}} e^{-2 \pi i h n / k} \int_{-\vartheta_{h, k}^{\prime}}^{\vartheta_{h, k}^{\prime \prime}} v_{d}\left(e^{(2 \pi i / k)(h+i z)}\right) e^{2 \pi n z / k} d \Phi .
$$

Lemma 10.1. Let $c_{h, k}(n):=(-12 / n) e^{(\pi i h / 12 k) n^{2}}$. Then if $h \neq 0$ then $c_{h, k}(n)$ is $24 k$ periodic and has mean value 0 .

Applying this lemma with the proposition in Section 3 of [Lawrence and Zagier 1999], we have the following.

Lemma 10.2. Let $q=e^{(2 \pi i / k)(h+i z)}$. Then

$$
\sum_{n=0}^{\infty}\left(\frac{-12}{n}\right) q^{n^{2} / 24} \sim \sum_{r=0}^{\infty} L(-2 r, h, k)\left(\frac{-\pi}{12 k}\right)^{r} \frac{z^{r}}{r !}
$$


where

$$
L(-r, h, k):=-\frac{(24 k)^{r}}{r+1} \sum_{n=1}^{24 k} c_{h, k}(n) B_{r+1}\left(\frac{n}{24 k}\right)
$$

where $B_{r}(x)$ denotes the $r$-th Bernoulli polynomial and $c_{k}$ is defined in Lemma 10.1. If $h=0$ we have

$$
L(-r):=L(-r, 0,1)=-\frac{6^{r}}{r+1}\left(B_{r+1}\left(\frac{1}{6}\right)-B_{r+1}\left(\frac{5}{6}\right)\right) .
$$

By (2-2) we have

$(-q)_{\infty}^{2}= \begin{cases}\frac{1}{2} e^{\frac{\pi}{12 k}\left(\frac{1}{z}+2 z\right)} i^{\frac{1-k}{2}} \omega_{h, k} e^{-\frac{\pi i h}{4 k}+\frac{\pi i k h}{4}}(h / k)\left(1+O\left(e^{-\frac{\pi}{k z}}\right)\right) & \text { if } 2 \nmid k, \\ O\left(e^{-\frac{\pi}{6 k z}}\right) & \text { if } 2 \mid k,\end{cases}$

so that we have the following results.

Proposition 10.3. Let $q=e^{(2 \pi i / k)(h+i z)}$ with $0 \leq h<k$ and $(h, k)=1$. If $2 \mid k$, then $v_{d}(q)+\sum_{n \geq 0}(-1)^{n} q^{(n+1)(n+2) / 2}=O\left(e^{-\pi /(6 k z)}\right)$. When $2 \nmid k$, we have

$$
\begin{aligned}
v_{d}(q)+ & \sum_{n \geq 0}(-1)^{n} q^{\frac{(n+1)(n+2)}{2}} \\
& \sim \frac{1}{2} e^{\frac{\pi}{12 k}\left(\frac{1}{z}+2 z\right)} i^{\frac{1-k}{2}} \omega_{h, k} e^{-\frac{\pi i h}{4 k}+\frac{\pi i k h}{4}}\left(\frac{h}{k}\right) \sum_{r=0}^{\infty} L(-2 r, k)\left(\frac{-\pi}{12 k}\right)^{r} \frac{z^{r}}{r !}
\end{aligned}
$$

Using these asymptotics with (10-1) and the integral evaluation

$$
\int_{-\vartheta_{h, k}^{\prime}}^{\vartheta_{h, k}^{\prime \prime}} e^{\frac{\pi}{12 k}\left(m z+\frac{1}{z}\right)} z^{\frac{1}{2}-j} d \Phi=\frac{2 \pi}{k} m^{\frac{j}{2}-\frac{3}{4}} I_{\frac{3}{2}-j}\left(\frac{\pi \sqrt{m}}{6 k}\right)+O\left(N^{-\frac{1}{4}}\right)
$$

(see [Lehner 1964] for details), we have

$$
\begin{aligned}
& V_{d}(n) \sim(-1)^{n+1} \delta(n) \\
& +\pi \sum_{\substack{2 \nmid k<N \\
(h, k)=1}} \frac{\mathscr{A}_{k}(n)}{k} \sum_{r=0}^{\infty} L(-2 r, k)\left(\frac{-\pi}{12 k}\right)^{r} \frac{1}{r !}(24 n+2)^{-\frac{r+1}{2}} I_{r-1}\left(\frac{\pi}{6 k} \sqrt{24 n+2}\right)
\end{aligned}
$$

where

$$
\begin{aligned}
& \mathscr{A}_{k}(n)=\sum_{(h, k)=1} i^{\frac{1-k}{2}}\left(\frac{h}{k}\right) \omega_{h, k} e^{-\frac{\pi i h}{4}\left(\frac{1}{k}+k\right)-\frac{2 \pi i h n}{k}}, \\
& \delta(m)= \begin{cases}1 & \text { if } m=(n+1)(n+2) / 2 \text { for some } n, \\
0 & \text { else. }\end{cases}
\end{aligned}
$$


The main term comes from $k=1$ and so we have

$$
\begin{aligned}
& V_{d}(n) \sim(-1)^{n+1} \delta(n) \\
& \quad+\pi \sum_{r=0}^{\infty} L(-2 r)\left(\frac{-\pi}{12}\right)^{r} \frac{1}{r !}(24 n+2)^{-\frac{r+1}{2}} I_{r-1}\left(\frac{\pi}{6} \sqrt{24 n+2}\right) \\
& \sim(-1)^{n+1} \delta(n)+\frac{\sqrt{3} e^{\frac{\pi}{6} \sqrt{24 n+2}}}{(24 n+2)^{\frac{3}{4}}} \sum_{r=0}^{\infty} L(-2 r)\left(\frac{\pi}{12}\right)^{r} \frac{(-1)^{r}}{r !} \\
& \quad \times \sum_{\ell=0}^{\infty} \frac{(-1)^{\ell}}{\ell !}\left(\frac{3}{4 \pi}\right)^{\ell} p(\ell, r)(24 n+2)^{-\frac{r+\ell}{2}} \\
& \sim(-1)^{n+1} \delta(n)+\frac{\sqrt{3} e^{\frac{\pi}{6}} \sqrt{24 n+2}}{(24 n+2)^{\frac{3}{4}}} \sum_{m=0}^{\infty} \frac{(-1)^{m}}{2^{2 m} m !(24 n+2)^{\frac{m}{2}}} \gamma(m) .
\end{aligned}
$$

\section{The $q$-series $v_{1}(q)$}

We have used a variety of different methods to establish the modular properties of the $q$-series defined by $v_{2}(q)$ or

$$
\sum_{n \neq 0} \frac{(-1)^{n+1} n q^{\left(n^{2}+n\right) / 2}}{1-q^{n}}
$$

On the other hand, we quoted the results of Bringmann [2008] for the modularity of $v_{1}(q)$. But this $q$-series is susceptible to similar methods of the ones discussed here. Analogous to Theorem 1.3 and Proposition 8.1 we have the following result for the Appell sum appearing in the definition in $v_{1}(q)$ and the Appell-like sum arising from the holomorphic projection operation.

Theorem 11.1. In the notation above,

$$
\begin{aligned}
\pi_{\mathrm{hol}}\left(\eta \eta^{*}\right) & =\sum_{n \neq 0}(-1)^{n}\left(\frac{-3}{n-1}\right) \frac{n q^{n(n+1) / 6}}{1-q^{n}} \\
& =\sum_{n \neq 0} \frac{(-1)^{n} q^{3 n(n+1) / 2}}{\left(1-q^{n}\right)^{2}}+\sum_{n=1}^{\infty} \frac{q^{n}}{\left(1-q^{n}\right)^{2}}=\sum_{n>0} \frac{(q ; q)_{n-1} q^{n}}{\left(1-q^{n}\right)} .
\end{aligned}
$$

Remark. It follows that

$$
\sum_{n=1}^{\infty} \operatorname{spt}(n) q^{n}=\frac{1}{(q)_{\infty}} \pi_{\mathrm{hol}}\left(\eta^{*} \eta\right)=\frac{1}{(q)_{\infty}} \sum_{n \neq 0}(-1)^{n}\left(\frac{-3}{n-1}\right) \frac{n q^{n(n+1) / 6}}{1-q^{n}}
$$


where $\operatorname{spt}(n)$ is the smallest parts function and is equal to the sum of the number of smallest parts in the partitions of $n$. See [Andrews 2008] for more on the spt-function.

Sketch of proof of Theorem 11.1. The first equality is given in Zagier's Bourbaki lecture [2009], while the second equality can be proved via "modular" methods discussed above. It would be interesting to establish a $q$-series proof of the second equality.

Finally, the last equality is derived in [Andrews 2008]. It may also be derived from the recent results of the first author, Garvan, and Liang [Andrews et al. 2012]. Namely, letting $z=1$ in [ibid., Theorem 2.4] gives

$$
\sum_{n \geq 1} \frac{(q)_{n-1} q^{n}}{\left(1-q^{n}\right)}=\sum_{n \neq 0} \frac{(-1)^{n-1} q^{n(n+1) / 2}}{\left(1-q^{n}\right)^{2}}-\sum_{n \neq 0} \frac{(-1)^{n-1} q^{n(3 n+1) / 2}}{\left(1-q^{n}\right)^{2}} .
$$

The result follows from the well known identity

$$
\sum_{n \neq 0} \frac{(-1)^{n-1} q^{n(n+1) / 2}}{\left(1-q^{n}\right)^{2}}=\sum_{n=1}^{\infty} \frac{q^{n}}{\left(1-q^{n}\right)^{2}} .
$$

\section{References}

[Andrews 1981] G. E. Andrews, "Ramanujan's "lost" notebook, I: Partial $\theta$-functions", Adv. in Math. 41:2 (1981), 137-172. MR 83m:10034a Zbl 0477.33001

[Andrews 1998] G. E. Andrews, The theory of partitions, Cambridge Mathematical Library, Cambridge University Press, 1998. MR 99c:11126 Zbl 0996.11002

[Andrews 2008] G. E. Andrews, "The number of smallest parts in the partitions of $n$ ", J. Reine Angew. Math. 624 (2008), 133-142. MR 2009m:11172 Zbl 1153.11053

[Andrews 2011] G. E. Andrews, “Concave compositions”, Electron. J. Combin. 18:2 (2011), Paper 6, 13. MR 2012e:05039 Zbl 1229.05029

[Andrews 2013] G. E. Andrews, "Concave and convex compositions”, Ramanujan J. 31:1-2 (2013), 67-82. MR 3048655 Zbl 06181374

[Andrews et al. 2012] G. E. Andrews, F. G. Garvan, and J. Liang, "Combinatorial interpretations of congruences for the spt-function”, Ramanujan J. 29:1-3 (2012), 321-338. MR 2994105 Zbl 06120459

[Bailey 1950] W. N. Bailey, "On the basic bilateral hypergeometric series ${ }_{2} \Psi_{2}$ ", Quart. J. Math., Oxford Ser. (2) 1 (1950), 194-198. MR 12,178e Zbl 0038.05001

[Bringmann 2008] K. Bringmann, "On the explicit construction of higher deformations of partition statistics”, Duke Math. J. 144:2 (2008), 195-233. MR 2009e:11203 Zbl 1154.11034

[Bringmann and Folsom 2013] K. Bringmann and A. Folsom, "On the asymptotic behavior of Kac-Wakimoto characters", Proc. Amer. Math. Soc. 141:5 (2013), 1567-1576. MR 3020844 Zbl 06146504

[Bringmann and Mahlburg 2011] K. Bringmann and K. Mahlburg, "An extension of the HardyRamanujan circle method and applications to partitions without sequences", Amer. J. Math. 133:4 (2011), 1151-1178. MR 2012g:11184 Zbl 1251.11072 
[Bringmann and Mahlburg 2013] K. Bringmann and K. Mahlburg, "Asymptotic formulas for coefficients of Kac-Wakimoto characters", Math. Proc. Cambridge Philos. Soc. 155:1 (2013), 51-72. MR 3065259 Zbl 06191889

[Bringmann et al. 2012] K. Bringmann, K. Mahlburg, and R. C. Rhoades, "Taylor coefficients of mockJacobi forms and moments of partition statistics”, preprint, 2012, Available at math.stanford.edu/ $\sim$ rhoades/FILES/RCZ.pdf.

[Bryson et al. 2012] J. Bryson, K. Ono, S. Pitman, and R. C. Rhoades, "Unimodal sequences and quantum and mock modular forms", Proc. Natl. Acad. Sci. USA 109:40 (2012), 16063-16067. MR 2994899

[Cheng 2010] M. C. N. Cheng, “ $K 3$ surfaces, $N=4$ dyons and the Mathieu group $M_{24}$ ”, Commun. Number Theory Phys. 4:4 (2010), 623-657. MR 2012e:11076 Zbl 1231.14031

[Choi 2011] Y.-S. Choi, "The basic bilateral hypergeometric series and the mock theta functions", Ramanujan J. 24:3 (2011), 345-386. MR 2012e:33042 Zbl 1225.33019

[Coates 1986] J. Coates, "The work of Gross and Zagier on Heegner points and the derivatives of L-series”, pp. 57-72 in Séminaire Bourbaki, 1984/85, Astérisque 133-134, Société Mathématique de France, Paris, 1986. MR 87j:11058 Zbl 0608.14020

[Diaconis et al. 2013] P. Diaconis, S. Janson, and R. C. Rhoades, "Note on a partition limit theorem for rank and crank", Bull. Lond. Math. Soc. 45:3 (2013), 551-553. MR 3065024 Zbl 06176878

[Eguchi and Hikami 2009] T. Eguchi and K. Hikami, "Superconformal algebras and mock theta functions, II: Rademacher expansion for K3 surface”, Commun. Number Theory Phys. 3:3 (2009), 531-554. MR 2011b:58048 Zbl 1189.81191

[Eguchi et al. 2011] T. Eguchi, H. Ooguri, and Y. Tachikawa, "Notes on the $K 3$ surface and the Mathieu group $M_{24}$ ”, Exp. Math. 20:1 (2011), 91-96. MR 2012e:58039

[Fine 1988] N. J. Fine, Basic hypergeometric series and applications, Mathematical Surveys and Monographs 27, American Mathematical Society, Providence, RI, 1988. MR 91j:33011 Zbl 0647.05004

[Fristedt 1993] B. Fristedt, "The structure of random partitions of large integers", Trans. Amer. Math. Soc. 337:2 (1993), 703-735. MR 93h:11090 Zbl 0795.05009

[Gasper and Rahman 2004] G. Gasper and M. Rahman, Basic hypergeometric series, 2nd ed., Encyclopedia of Mathematics and its Applications 96, Cambridge University Press, 2004. MR 2006d:33028 Zbl 1129.33005

[Gross and Zagier 1986] B. H. Gross and D. B. Zagier, "Heegner points and derivatives of $L$-series", Invent. Math. 84:2 (1986), 225-320. MR 87j:11057 Zbl 0608.14019

[Heubach and Mansour 2010] S. Heubach and T. Mansour, Combinatorics of compositions and words, Discrete Mathematics and its Applications, CRC Press, Boca Raton, FL, 2010. MR 2010i:68122 Zbl 1184.68373

[Lawrence and Zagier 1999] R. Lawrence and D. Zagier, "Modular forms and quantum invariants of 3-manifolds", Asian J. Math. 3:1 (1999), 93-107. MR 2000j:11057 Zbl 1024.11028

[Lehner 1964] J. Lehner, "On automorphic forms of negative dimension", Illinois J. Math. 8 (1964), 395-407. MR 29 \#2230 Zbl 0129.05801

[MacMahon 1893] P. A. MacMahon, "Memoir on the theory of compositions of numbers", London R. S. Phil. A 184 (1893), 835-901. JFM 25.0258.01

[Malmendier and Ono 2012] A. Malmendier and K. Ono, "Moonshine and Donaldson invariants of $\mathbb{C} P^{2}$,, preprint, 2012. arXiv 1207.5139

[Ono 2004] K. Ono, The web of modularity: Arithmetic of the coefficients of modular forms and $q$-series, CBMS Regional Conference Series in Mathematics 102, Amer. Math. Soc., Providence, 2004. MR 2005c:11053 Zbl 1119.11026 
[Ono 2008] K. Ono, "Mock theta functions, ranks, and Maass forms", pp. 119-141 in Surveys in number theory, edited by K. Alladi, Dev. Math. 17, Springer, New York, 2008. MR 2009j:11170 Zbl 1183.11064

[Ono 2009] K. Ono, "Unearthing the visions of a master: harmonic Maass forms and number theory", pp. 347-454 in Current developments in mathematics, 2008, edited by D. Jerison et al., International Press, 2009. MR 2010m:11060 Zbl 1229.11074

[Pittel 1997] B. Pittel, “On a likely shape of the random Ferrers diagram”, Adv. in Appl. Math. 18:4 (1997), 432-488. MR 98m:60013 Zbl 0894.11039

[Rhoades 2012] R. C. Rhoades, "Strongly unimodal sequences and mixed mock modular forms", preprint, 2012, Available at math.stanford.edu/ rhoades/FILES/unimodal.pdf.

[Rhoades 2013] R. C. Rhoades, "Families of quasimodular forms and Jacobi forms: the crank statistic for partitions", Proc. Amer. Math. Soc. 141:1 (2013), 29-39. MR 2988708

[Shimura 1973] G. Shimura, "On modular forms of half integral weight”, Ann. of Math. (2) 97 (1973), 440-481. MR 48 \#10989 Zbl 0266.10022

[Sturm 1980] J. Sturm, "Projections of $C^{\infty}$ automorphic forms", Bull. Amer. Math. Soc. (N.S.) 2:3 (1980), 435-439. MR 81g:10039 Zbl 0433.10013

[Vershik 1995] A. M. Vershik, "Asymptotic combinatorics and algebraic analysis", pp. 1384-1394 in Proceedings of the International Congress of Mathematicians (Zürich, 1994), vol. 2, edited by S. D. Chatterji, Birkhäuser, Basel, 1995. MR 98e:60018 Zbl 0843.05003

[Zagier 2001] D. Zagier, "Vassiliev invariants and a strange identity related to the Dedekind etafunction", Topology 40:5 (2001), 945-960. MR 2002g:11055 Zbl 0989.57009

[Zagier 2009] D. Zagier, "Ramanujan's mock theta functions and their applications (after Zwegers and Ono-Bringmann)”, pp. 143-164 (exposé 986) in Séminaire Bourbaki 2007/2008, Astérisque 326, Société Mathématique de France, Paris, 2009. MR 2011h:11049 Zbl 1198.11046

[Zwegers 2002] S. Zwegers, Mock theta functions, Ph.D. thesis, Universiteit Utrecht, 2002, Available at igitur-archive.library.uu.nl/dissertations/2003-0127-094324/full.pdf.

Communicated by Georgia Benkart

Received 2012-07-30 Revised 2012-09-10 Accepted 2012-10-22

andrews@math.psu.edu

Department of Mathematics,

The Pennsylvania State University, 109 McAllister Building, University Park PA 16802-7000, United States

rhoades@math.stanford.edu Department of Mathematics, Stanford University, Bldg 380, Stanford CA 94305, United States

szwegers@uni-koeln.de

Mathematical Institute, University of Cologne, Weyertal 86-90, D-50931 Cologne, Germany 


\section{Algebra \& Number Theory}

msp.org/ant

\section{EDITORS}

MANAGING EDITOR

Bjorn Poonen

Massachusetts Institute of Technology

Cambridge, USA

\author{
EDITORIAL BOARD CHAIR \\ David Eisenbud \\ University of California \\ Berkeley, USA
}

\section{BOARD OF EDITORS}

Georgia Benkart

Dave Benson

Richard E. Borcherds

John H. Coates

J-L. Colliot-Thélène

Brian D. Conrad

Hélène Esnault

Hubert Flenner

Edward Frenkel

Andrew Granville

Joseph Gubeladze

Roger Heath-Brown

Ehud Hrushovski

Craig Huneke

Mikhail Kapranov

Yujiro Kawamata

János Kollár

Yuri Manin

Barry Mazur

Philippe Michel
University of Wisconsin, Madison, USA

University of Aberdeen, Scotland

University of California, Berkeley, USA

University of Cambridge, UK

CNRS, Université Paris-Sud, France

University of Michigan, USA

Freie Universität Berlin, Germany

Ruhr-Universität, Germany

University of California, Berkeley, USA

Université de Montréal, Canada

San Francisco State University, USA

Oxford University, UK

Hebrew University, Israel

University of Virginia, USA

Yale University, USA

University of Tokyo, Japan

Princeton University, USA

Northwestern University, USA

Harvard University, USA

École Polytechnique Fédérale de Lausanne
Susan Montgomery

Shigefumi Mori

Raman Parimala

Jonathan Pila

Victor Reiner

Karl Rubin

Peter Sarnak

Joseph H. Silverman

Michael Singer

Vasudevan Srinivas

J. Toby Stafford

Bernd Sturmfels

Richard Taylor

Ravi Vakil

Michel van den Bergh

Marie-France Vignéras

Kei-Ichi Watanabe

Efim Zelmanov

Shou-Wu Zhang
University of Southern California, USA

RIMS, Kyoto University, Japan

Emory University, USA

University of Oxford, UK

University of Minnesota, USA

University of California, Irvine, USA

Princeton University, USA

Brown University, USA

North Carolina State University, USA

Tata Inst. of Fund. Research, India

University of Michigan, USA

University of California, Berkeley, USA

Harvard University, USA

Stanford University, USA

Hasselt University, Belgium

Université Paris VII, France

Nihon University, Japan

University of California, San Diego, USA

Princeton University, USA

PRODUCTION

production@msp.org

Silvio Levy, Scientific Editor

See inside back cover or msp.org/ant for submission instructions.

The subscription price for 2013 is US $\$ 200 /$ year for the electronic version, and $\$ 350 /$ year $(+\$ 40$, if shipping outside the US) for print and electronic. Subscriptions, requests for back issues and changes of subscribers address should be sent to MSP.

Algebra \& Number Theory (ISSN 1944-7833 electronic, 1937-0652 printed) at Mathematical Sciences Publishers, 798 Evans Hall \#3840, c/o University of California, Berkeley, CA 94720-3840 is published continuously online. Periodical rate postage paid at Berkeley, CA 94704, and additional mailing offices.

ANT peer review and production are managed by EditFLOW ${ }^{\circledR}$ from Mathematical Sciences Publishers.

\section{PUBLISHED BY}

- mathematical sciences publishers

nonprofit scientific publishing

http://msp.org/

(C) 2013 Mathematical Sciences Publishers 


\section{Algebra \& Number Theory}

Volume $7 \quad$ No. $9 \quad 2013$

Multiplicities associated to graded families of ideals Steven Dale Cutkosky

Normal coverings of linear groups

JOHN R. BRITNELL and ATTILA MARÓTI

Modularity of the concave composition generating function

George E. ANDrews, Robert C. RhOAdes and SANDER P. ZWEgERS

ANDREW NILES

Regular permutation groups of order $m p$ and Hopf Galois structures

2203

ТIMOTHY KOHL

Further evidence for conjectures in block theory

2241

BENJAMIN SAMBALE

Network parametrizations for the Grassmannian

2275

Kelli TALASKa and LAUREN Williams

Chow quotients of toric varieties as moduli of stable log maps

2313

Qile Chen and Matthew Satriano

Vinberg's representations and arithmetic invariant theory

JACK A. THORNE 